\title{
A theory of inner Riesz balayage and its applications
}

by

\author{
Natalia ZORII \\ Presented by the Editors \\ Dedicated to Professor Wiestaw Pleśniak \\ on the occasion of his 75th birthday
}

\begin{abstract}
Summary. We establish a theory of balayage for the Riesz kernel $|x-y|^{\alpha-n}, \alpha \in(0,2]$, on $\mathbb{R}^{n}, n \geq 3$, alternative to that suggested in the book by Landkof. A need for that is caused by the fact that the balayage in that book is defined by means of the integral representation, which, however, so far is not completely justified. Our alternative approach is mainly based on Cartan's ideas concerning inner balayage, formulated by him for the Newtonian kernel. Applying the theory of inner Riesz balayage thereby developed, we obtain a number of criteria for the existence of an inner equilibrium measure $\gamma_{A}$ for $A \subset \mathbb{R}^{n}$ arbitrary, in particular given in terms of the total mass of the inner swept measure $\mu^{A}$ with $\mu$ suitably chosen. For example, $\gamma_{A}$ exists if and only if $\varepsilon^{A^{*}} \neq \varepsilon$, where $\varepsilon$ is the Dirac measure at $x=0$ and $A^{*}$ the inverse of $A$ relative to the sphere $|x|=1$, which leads to a Wiener type criterion of inner $\alpha$-irregularity. The results obtained are illustrated by examples.
\end{abstract}

1. Introduction. A major goal of our study is to establish a theory of balayage for the Riesz kernel $|x-y|^{\alpha-n}, \alpha \in(0,2]$, on $\mathbb{R}^{n}, n \geq 3$, alternative to that suggested in [11, Chapter IV, Section $6, \mathrm{n}^{\circ} 25$ and Chapter V, Section $1, \mathrm{n}^{\circ} 2$ ]. A need for that is caused by the fact that the balayage $\mu^{A}$ of a positive Radon measure $\mu$ on $\mathbb{R}^{n}$ to a Borel set $A \subset \mathbb{R}^{n}$ is defined in [11] by means of the integral representation

$$
\mu^{A}=\int \varepsilon_{y}^{A} d \mu(y)
$$

2020 Mathematics Subject Classification: Primary 31C15.

Key words and phrases: inner Riesz balayage, inner Riesz equilibrium measure, Wiener type criterion of inner $\alpha$-irregularity, $\mu$-adequate family of Radon measures.

Received 4 November 2019; revised 30 January 2020.

Published online 2 March 2020. 
where $\varepsilon_{y}$ is the unit Dirac measure at $y \in \mathbb{R}^{n}$. However, this requires that the family $\left(\varepsilon_{y}^{A}\right)_{y \in \mathbb{R}^{n}}$ be $\mu$-adequate in the sense of [3, Chapter V, Section 3, $\mathrm{n}^{\circ} 1$, Definition 1]. As pointed out in [3, Chapter V, Section 3, $\mathrm{n}^{\circ} 1$, Remark], it is not enough to verify that for every $f \in C_{0}\left(\mathbb{R}^{n}\right)$, the function $y \mapsto \int f d \varepsilon_{y}^{A}$ is $\mu$-measurable on $\mathbb{R}^{n}$ (as is done in [11, p. 214, footnote 12]); see also counterexamples (without $\mu$-adequacy) in [3, Chapter V, Section 3, Exercises 1, 2]. Here $C_{0}\left(\mathbb{R}^{n}\right)$ is the class of all finite continuous functions on $\mathbb{R}^{n}$ with compact support.

For $A$ closed and $\mu$ carried by $A^{c}:=\mathbb{R}^{n} \backslash A$, the $\mu$-adequacy of the family $\left(\varepsilon_{y}^{A}\right)_{y \in A^{c}}$, and hence the validity of the integral representation 1.1 , has recently been proven in [10, Lemma 3.16, Theorem 3.17]. Theorem 8.2 below strengthens [10, Theorem 3.17] to $A$ arbitrary and $\mu$ carried by $A^{c}$, the concept of balayage being now understood in the sense described in Section 3 . However, the question whether the integral representation (1.1) holds for any $\mu$ is still open.

Leaving aside the question of validity of (1.1), we establish instead an alternative theory of Riesz balayage by generalizing H. Cartan's [6] ideas concerning inner balayage, formulated for the Newtonian kernel $|x-y|^{2-n}$. To briefly explain the results obtained, we need the following notions and notation.

Let $\mathfrak{M}$ denote the linear space of all (signed) Radon measures $\nu$ on $\mathbb{R}^{n}$, equipped with the vague topology, i.e. the topology of pointwise convergence on $C_{0}\left(\mathbb{R}^{n}\right)$. Given $\nu, \nu_{1} \in \mathfrak{M}$, we define the potential and the mutual energy by

$$
\begin{aligned}
U^{\nu}(x) & :=\int|x-y|^{\alpha-n} d \nu(y), \quad x \in \mathbb{R}^{n}, \\
E\left(\nu, \nu_{1}\right) & :=\int|x-y|^{\alpha-n} d\left(\nu \otimes \nu_{1}\right)(x, y),
\end{aligned}
$$

respectively (provided, of course, that the corresponding right-hand side is well defined as a finite number or $\pm \infty)$. For $\nu=\nu_{1}, E\left(\nu, \nu_{1}\right)$ defines the energy $E(\nu):=E(\nu, \nu)$ of $\nu$. All $\nu \in \mathfrak{M}$ with $E(\nu)$ finite form a pre-Hilbert space $\mathcal{E}$ with the inner product $\left(\nu, \nu_{1}\right):=E\left(\nu, \nu_{1}\right)$ and the norm $\|\nu\|:=$ $\sqrt{E(\nu)}$. The topology on $\mathcal{E}$ defined by $\|\cdot\|$ is said to be strong.

For an arbitrary set $Q \subset \mathbb{R}^{n}$, we denote by $\mathfrak{M}_{Q}^{+}$the cone of all positive $\nu \in \mathfrak{M}$ carried by $Q$, which means that $Q$ is $\nu$-measurable and $Q^{c}$ is $\nu$ negligible. Write $\mathcal{E}_{Q}^{+}:=\mathcal{E} \cap \mathfrak{M}_{Q}^{+}, \mathfrak{M}^{+}:=\mathfrak{M}_{\mathbb{R}^{n}}^{+}$, and $\mathcal{E}^{+}:=\mathcal{E}_{\mathbb{R}^{n}}^{+}$.

To establish the theory of inner Riesz balayage, we first consider $\mu$ with finite energy, and we define the inner balayage $\mu^{A} \in \mathcal{E}^{+}$of $\mu \in \mathcal{E}^{+}$to $A \subset \mathbb{R}^{n}$ arbitrary as the strong and vague limit of $\mu^{K}$ as $K$ increases along the upper directed family $\mathfrak{C}_{A}$ of all compact subsets of $A$ (see Theorem 3.4), where $\mu^{K}$ is the orthogonal projection of $\mu$ in the pre-Hilbert space $\mathcal{E}$ onto the strongly complete convex cone $\mathcal{E}_{K}^{+}$(cf. Theorem 3.1). Alternatively, this $\mu^{A}$ is, in fact, the orthogonal projection of $\mu$ onto the strong closure of $\mathcal{E}_{A}^{+}$ (see Theorem 3.4). 
Observing that

$$
E\left(\mu, \lambda^{A}\right)=E\left(\mu^{A}, \lambda\right) \quad \text { for all } \lambda \in \mathcal{E}^{+},
$$

we now define the inner balayage $\mu^{A} \in \mathfrak{M}^{+}$of $\mu \in \mathfrak{M}^{+}$to $A$ as a measure satisfying this symmetry identity (Definition 3.9$)\left({ }^{1}\right)$. This $\mu^{A}$ exists, is unique, and can equivalently be determined by either of the following two limit relations:

$$
\mu_{k}^{A} \rightarrow \mu^{A} \quad \text { vaguely, } \quad U^{\mu_{k}^{A} \uparrow} U^{\mu^{A}} \text { pointwise on } \mathbb{R}^{n},
$$

where $\left(\mu_{k}\right) \subset \mathcal{E}^{+}$is any given sequence (net) such that $U^{\mu_{k}} \uparrow U^{\mu}$ pointwise on $\mathbb{R}^{n}$ (see Theorem 3.10 and its proof).

We emphasize that although $U^{\mu^{A}}=U^{\mu}$ n.e. on $A$, that is, everywhere on $A$ except for a set of zero inner capacity $c_{\alpha}(\cdot)$ (see Theorem 3.10), this property no longer characterizes $\mu^{A}$ uniquely (as it does for $A$ closed and $\mu \in \mathcal{E}^{+}$, cf. Theorem 3.1, which is illustrated in Remark 3.12. Nevertheless this uniqueness does hold whenever $A$ is closed and $\mu \in \mathfrak{M}^{+}$is carried by $A^{c}$ (see Corollary 8.4).

Further, we apply the concept of inner Riesz balayage thus introduced to the problem of existence of an inner equilibrium measure $\gamma_{A}$ for $A$ arbitrary. This $\gamma_{A}$ can be defined e.g. as the vague limit of $\gamma_{K}$ as $K$ increases along $\mathfrak{C}_{A}$, while the equilibrium measure $\gamma_{K}$ on $K$ compact is defined as usual (see e.g. [11, Chapter II, Section 2, $\left.\left.\mathrm{n}^{\circ} 7\right]\right)$.

For $A$ Borel, necessary and sufficient conditions for the existence of $\gamma_{A}$ have been provided in [11, Theorem 5.1]. However, [11, Theorem 5.1] has not been completely justified, because the proof of its necessity part is based on the concept of balayage, introduced in [11, Chapter IV, Section 6, $\left.\mathrm{n}^{\circ} 25\right]$ with the aid of the integral representation (1.1).

By use of our concept of inner Riesz balayage, we fix that gap in [11, proof of Theorem 5.1], and moreover we strengthen [11, Theorem 5.1] to $A$ arbitrary (see Theorem 5.5). Observing that the existence of $\gamma_{A}$ does not necessarily imply the finiteness of $c_{\alpha}(A)$, we illustrate this by means of Example 5.8 .

An inner $\alpha$-irregular point $y$ for $A$ is defined by the relations $y \in \bar{A}$ and $\varepsilon_{y}^{A} \neq \varepsilon_{y}$. We show that $\varepsilon_{y}^{A} \neq \varepsilon_{y}$ is equivalent to the existence of an inner equilibrium measure $\gamma_{A^{*}}$ for the inverse $A^{*}$ of $A \backslash\{y\}$ relative to the sphere $S(y, 1)$; and then $\varepsilon_{y}^{A}$ is actually the Kelvin transform of $\gamma_{A^{*}}$ (Theorem 6.10. Combining Theorem 6.10 with Theorem 5.5 results in a Wiener type criterion of inner $\alpha$-irregularity (Theorem 6.4).

Other necessary and sufficient conditions for the existence of $\gamma_{A}$, now given in terms of the total mass $\mu^{A}\left(\mathbb{R}^{n}\right)$ with $\mu$ suitably chosen, are pro-

$\left({ }^{1}\right)$ The term 'inner balayage' is justified by showing that $\mu^{K} \rightarrow \mu^{A}$ vaguely as $K$ increases along $\mathfrak{C}_{A}$ (see Theorem 4.5). 
vided by Theorems 8.6 and 8.7 . It is shown that $\gamma_{A}$ exists whenever there is $\mu \in \mathfrak{M}_{A^{c}}^{+}$with

$$
\mu^{A}\left(\mathbb{R}^{n}\right)<\mu\left(\mathbb{R}^{n}\right),
$$

while for $A$ closed, this can be reversed (see Section 8.3 for further details). The proofs of Theorems 8.6 and 8.7 are based on Theorem 6.10 as well as on Theorem 8.2, establishing the integral representation (1.1) for $A$ arbitrary and $\mu$ carried by $\bar{A}^{c}$ and thereby strengthening [10, Theorem 3.17]. Theorems 8.6 and 8.7 are illustrated by Example 8.8 .

It is worth mentioning that the concept of inner Riesz balayage, defined in our study, differs from the concept of balayage by Brelot [5] as well as from that by Bliedtner and Hansen [1. The last two concepts are relevant to the concept of outer balayage (cf. [6]), which can be seen e.g. by comparing [5, Theorem IX.10] and [1, Chapter VI, Proposition 2.2] with our Theorem 6.4 and Corollary 4.6, respectively.

2. Preliminaries. This paper deals with the Riesz kernel $|x-y|^{\alpha-n}$ of order $0<\alpha \leq 2$ on $\mathbb{R}^{n}, n \geq 3$. In what follows we shall tacitly use the notions and notation introduced in Section 1

For $Q \subset \mathbb{R}^{n}$, let $\partial Q$ and $\bar{Q}$ denote the boundary and the closure of $Q$ in the Euclidean topology on $\mathbb{R}^{n}$. Write $B(y, r):=\left\{x \in \mathbb{R}^{n}:|x-y|<r\right\}$, where $r>0$, and let $S(y, r)$ and $\bar{B}(y, r)$ stand for the boundary and the closure of $B(y, r)$ in $\mathbb{R}^{n}$.

In this section we have gathered some basic facts of Riesz potential theory, often used below. When speaking of a measure $\mu \in \mathfrak{M}^{+}$, we always tacitly assume that $U^{\mu}$ is not identically infinite, or equivalently [11, Chapter I, Section $\left.3, \mathrm{n}^{\circ} 7\right]$

$$
\int_{|y|>1} \frac{d \mu(y)}{|y|^{n-\alpha}}<\infty .
$$

Then $U^{\mu}, \mu \in \mathfrak{M}^{+}$, is $\alpha$-superharmonic (hence lower semicontinuous (l.s.c.)) on $\mathbb{R}^{n}\left[11\right.$, Chapter I, Section $\left.6, \mathrm{n}^{\circ} 20\right]$, which is crucial to Theorems 2.1 and 2.4 below.

THEOREM 2.1. If an upper directed family $\left(U^{\mu_{t}}\right)_{t \in T}$, where $\mu_{t} \in \mathfrak{M}^{+}$for all $t \in T$, is majorized by $U^{\mu}$ with some $\mu \in \mathfrak{M}^{+}$, then there exists $\nu \in \mathfrak{M}^{+}$ such that $U^{\mu_{t}} \uparrow U^{\nu}$ pointwise on $\mathbb{R}^{n}$ and $\mu_{t} \rightarrow \nu$ vaguely (as $t$ increases along $T)$.

For $T$ countable, Theorem 2.1 is [11, Theorem 3.9]. The proof of [11, Theorem 3.9] can be generalized to $T$ uncountable with the aid of [7, Appendix VIII, Theorem 2] and [3, Chapter IV, Section 1, Theorem 1]. 
The Riesz kernel is strictly positive definite, that is, $E(\nu) \geq 0$ for every $\nu \in \mathfrak{M}$ (whenever $E(\nu)$ is well defined) and $E(\nu)=0$ only for $\nu=0$. Furthermore, it is perfect [9] in the sense that every strong Cauchy sequence (net) in $\mathcal{E}^{+}$converges strongly to any of its vague limit points, and the strong topology on $\mathcal{E}^{+}$is finer (stronger) than the vague topology on $\mathcal{E}^{+}$. Since any strongly bounded part of $\mathcal{E}^{+}$is vaguely bounded [9, Lemma 2.5.1], the cone $\mathcal{E}^{+}$is strongly complete. Hence, so is $\mathcal{E}_{F}^{+}$for $F \subset \mathbb{R}^{n}$ closed, the cone $\mathfrak{M}_{F}^{+}$ being vaguely closed.

For any $Q \subset \mathbb{R}^{n}$, the inner $\alpha$-Riesz capacity $c_{\alpha}(Q)$ is given by $\left({ }^{2}\right)$

$$
c_{\alpha}(Q):=1 / \inf E(\mu),
$$

where $\mu$ ranges over all $\mu \in \mathcal{E}_{Q}^{+}$with $\mu\left(\mathbb{R}^{n}\right)=1$. Then

$$
c_{\alpha}(K) \uparrow c_{\alpha}(Q) \quad \text { as } K \uparrow Q,
$$

where the abbreviation ' $K \uparrow Q$ ' means that $K$ increases along $\mathfrak{C}_{Q}$.

Lemma 2.2 (see [9, Lemma 2.3.1]). For any $Q \subset \mathbb{R}^{n}$,

$$
c_{\alpha}(Q)=0 \Longleftrightarrow \mathcal{E}_{Q}^{+}=\{0\} .
$$

A measure $\mu \in \mathfrak{M}^{+}$is said to be bounded if $\mu\left(\mathbb{R}^{n}\right)<\infty$, and $c_{\alpha}$-absolutely continuous if $\mu(K)=0$ for every compact $K \subset \mathbb{R}^{n}$ with $c_{\alpha}(K)=0$. It follows from Lemma 2.2 that any $\mu \in \mathcal{E}^{+}$is $c_{\alpha}$-absolutely continuous, but not conversely [11, pp. 134-135].

An assertion $\mathcal{U}$ is said to hold nearly everywhere (n.e.) on $Q \subset \mathbb{R}^{n}$ if the set of $x \in Q$ for which $\mathcal{U}(x)$ fails has zero inner capacity.

The following assertion amounts to the countable subadditivity of inner capacity in the form stated in [9, p. 158, Remark].

Lemma 2.3. Let $Q \subset \mathbb{R}^{n}$ be arbitrary, and $E_{k} \subset \mathbb{R}^{n}, k \in \mathbb{N}$, Borel. If an assertion $\mathcal{U}$ holds n.e. on $Q \cap E_{k}$ for every $k$, then $\mathcal{U}$ holds n.e. on the union of all $Q \cap E_{k}$.

The property of the Riesz kernel of order $\alpha \in(0,2]$, presented in the following assertion (see [11, Theorems 1.27, 1.29]), is known as the complete maximum principle; for $q=0$, it is also called the domination principle, and for $\nu=0$, the Frostman maximum principle.

TheOrem 2.4. If $U^{\mu} \leq U^{\nu}+q \mu$-a.e., where $\mu \in \mathcal{E}^{+}, \nu \in \mathfrak{M}^{+}$, and $q \in[0, \infty)$, then this inequality holds on all of $\mathbb{R}^{n}$.

Let $\mathcal{M} \subset \mathcal{E}^{+}$be a strongly closed convex cone containing $\mu=0$. Since then $\mathcal{M}$ is strongly complete, the following theorem is a particular case of [8, Theorem 1.12.3, Proposition 1.12.4(2)].

$\left({ }^{2}\right)$ The infimum over the empty set is taken to be $+\infty$. We also put $1 /(+\infty)=0$ and $1 / 0=+\infty$. 
Theorem 2.5. For any $\mu \in \mathcal{E}^{+}$, there is a unique $P \mu=P_{\mathcal{M}}(\mu) \in \mathcal{M}$ such that

$$
\|\mu-P \mu\|=\inf _{\nu \in \mathcal{M}}\|\mu-\nu\|=: \varrho(\mu, \mathcal{M}) .
$$

This $P \mu$ is called the orthogonal projection of $\mu$ in the pre-Hilbert space $\mathcal{E}$ onto $\mathcal{M}$, and it is characterized uniquely by the relations

$$
\begin{aligned}
& (\mu-P \mu, \lambda) \leq 0 \quad \text { for all } \lambda \in \mathcal{M}, \\
& (\mu-P \mu, P \mu)=0 .
\end{aligned}
$$

3. Inner Riesz balayage. Unless explicitly stated otherwise, in what follows we assume that $A$ is an arbitrary proper subset of $\mathbb{R}^{n}$ with $c_{\alpha}(A)>0$.

The notion of inner Riesz balayage of $\mu \in \mathfrak{M}^{+}$to $A$ will be defined in three steps, presented respectively in Sections 3.1, 3.2, and 3.3.

3.1. Step 1: $\mu \in \mathcal{E}^{+}$and $A$ closed. Assume first that $\mu \in \mathfrak{M}^{+}$has finite energy and $A$ is closed in $\mathbb{R}^{n}$.

Theorem 3.1. For $\mu \in \mathcal{E}^{+}$and $A$ closed, there exists $\mu^{A} \in \mathcal{E}_{A}^{+}$such that

$$
\begin{array}{ll}
U^{\mu^{A}}=U^{\mu} & \text { n.e. on } A, \\
U^{\mu^{A}} \leq U^{\mu} & \text { on } \mathbb{R}^{n} .
\end{array}
$$

This $\mu^{A}$ is actually the orthogonal projection of $\mu$ in the pre-Hilbert space $\mathcal{E}$ onto the convex cone $\mathcal{E}_{A}^{+}$, and it is uniquely determined within $\mathcal{E}_{A}^{+}$by (3.1).

Proof. For $A$ closed, the convex cone $\mathcal{E}_{A}^{+}$is strongly closed, because the strong topology on $\mathcal{E}^{+}$is stronger than the vague topology on $\mathcal{E}^{+}$while $\mathfrak{M}_{A}^{+}$is vaguely closed. According to Theorem 2.5. there exists therefore a unique orthogonal projection $P \mu=P_{\mathcal{E}_{A}^{+}}(\mu)$ of $\mu$ onto $\mathcal{E}_{A}^{+}$, and it is uniquely determined by 2.3 and 2.4 with $\mathcal{M}:=\mathcal{E}_{A}^{+}$. Relations 3.1 and 3.2 with $\mu^{A}:=P \mu$ can now be established in a manner similar to that in 11, proof of Theorem 4.16].

Indeed, the restriction $\left.\lambda\right|_{E}$ of any $\lambda \in \mathcal{E}^{+}$to the Borel set $E:=\{x \in A$ : $\left.U^{\mu}(x)>U^{P \mu}(x)\right\}$ belongs to $\mathcal{E}_{A}^{+}$, hence $\left(\mu-P \mu,\left.\lambda\right|_{E}\right) \leq 0$ by 2.3 , and consequently $\left.\lambda\right|_{E}=0$. Since $\lambda \in \mathcal{E}^{+}$has been chosen arbitrarily, $c_{\alpha}(E)=0$ according to Lemma 2.2. Thus,

$$
U^{P \mu} \geq U^{\mu} \quad \text { n.e. on } A \text {. }
$$

Another use of Lemma 2.2 now gives $U^{P \mu} \geq U^{\mu} P \mu$-a.e., which together with 2.4 shows that, actually, $U^{P \mu}=U^{\mu} P \mu$-a.e. By the domination principle (see Theorem 2.4), this yields (3.2), which combined with 3.3 establishes (3.1).

If 3.1) also holds with $\theta \in \mathcal{E}_{A}^{+}$, then $\nu:=\theta-P \mu \in \mathcal{E}$, and furthermore $U^{\nu}=0$ n.e. on $A$, hence $\nu$-a.e., again by Lemma 2.2 . We therefore obtain by 
integration $\|\nu\|=0$, which implies $\theta=P \mu$, the Riesz kernel being strictly positive definite.

REMARK 3.2. One could equally well write 'q.e.' (quasi everywhere) instead of 'n.e.' in (3.1), where 'q.e.' refers to outer capacity [11, Chapter II, Section $\left.2, \mathrm{n}^{\circ} 6\right]$. Indeed, $\psi:=U^{\mu^{A}}-U^{\mu}$, being the difference between two l.s.c. functions, is Borel measurable, and hence $\{x \in A: \psi(x) \neq 0\}$ is capacitable [11, Theorem 2.8].

Corollary 3.3. For any closed subset $F$ of $A$,

$$
\mu^{F}=\left(\mu^{A}\right)^{F} \quad \text { for every } \mu \in \mathcal{E}^{+} .
$$

Proof. According to Theorem 3.1, both $\mu^{F}$ and $\left(\mu^{A}\right)^{F}$ belong to $\mathcal{E}_{F}^{+}$, and moreover

$$
U^{\left(\mu^{A}\right)^{F}}=U^{\mu^{A}}=U^{\mu}=U^{\mu^{F}} \quad \text { n.e. on } F .
$$

Since (3.1) with $A=F$ determines $\mu^{F}$ uniquely within $\mathcal{E}_{F}^{+}$, 3.4 follows.

3.2. Step 2: $\mu \in \mathcal{E}^{+}$and $A$ arbitrary. Still requiring that $\mu \in \mathfrak{M}^{+}$ have finite energy, we now extend our analysis to $A$ arbitrary. Let $\mathcal{E}_{A}^{\prime}$ denote the strong closure of $\mathcal{E}_{A}^{+}$. Obviously, $\mathcal{E}_{A}^{\prime}$ is a strongly closed convex cone in $\mathcal{E}^{+}$.

Theorem 3.4. For $\mu \in \mathcal{E}^{+}$and $A$ arbitrary, there is a unique $\mu^{A} \in \mathcal{E}^{+}$ such that

$$
\mu^{K} \rightarrow \mu^{A} \quad \text { strongly and vaguely in } \mathcal{E}^{+},
$$

where $K$ increases along the upper directed family $\mathfrak{C}=\mathfrak{C}_{A}$ of all compact subsets of $A$ and $\mu^{K}$ is defined in Theorem 3.1. This $\mu^{A}$ can alternatively be defined as the orthogonal projection of $\mu$ onto $\mathcal{E}_{A}^{\prime}$, that is $\left(^{3}\right)$.

$$
\left\|\mu-\mu^{A}\right\|=\min _{\nu \in \mathcal{E}_{A}^{\prime}}\|\mu-\nu\|=\varrho\left(\mu, \mathcal{E}_{A}^{\prime}\right) .
$$

Proof. In view of (2.2) and our assumption $c_{\alpha}(A)>0$, we may consider only those $K \in \mathfrak{C}$ whose capacity is $>0$. Since obviously

$$
\left\|\mu-\mu^{K^{\prime}}\right\| \leq\left\|\mu-\mu^{K}\right\| \quad \text { whenever } K \subset K^{\prime} \quad\left(\text { where } K, K^{\prime} \in \mathfrak{C}\right),
$$

Lemma 4.1.1 in [9] with $\mathcal{H}:=\mathcal{E}, \Gamma:=\left\{\mu-\nu: \nu \in \mathcal{E}_{K^{\prime}}^{+}\right\}$, and $\lambda:=\mu-\mu^{K^{\prime}}$ yields

$$
\left\|\mu^{K}-\mu^{K^{\prime}}\right\|^{2}=\left\|\left(\mu-\mu^{K}\right)-\left(\mu-\mu^{K^{\prime}}\right)\right\|^{2} \leq\left\|\mu-\mu^{K}\right\|^{2}-\left\|\mu-\mu^{K^{\prime}}\right\|^{2} .
$$

Being decreasing and lower bounded, the net $\left(\left\|\mu-\mu^{K}\right\|\right)_{K \in \mathfrak{C}}$ is Cauchy in $\mathbb{R}$, which together with the last display implies that the net $\left(\mu^{K}\right)_{K \in \mathfrak{C}}$ is strong Cauchy in $\mathcal{E}^{+}$. Being thus strongly bounded, $\left(\mu^{K}\right)_{K \in \mathfrak{C}}$ is vaguely bounded by [9, Lemma 2.5.1], and has a vague limit point $\mu_{0} \in \mathfrak{M}^{+}$according to

$\left({ }^{3}\right)$ This implies that for $A$ closed, the measure $\mu^{A}$ determined by Theorem 3.4 coincides with that determined by Theorem 3.1 
[3. Chapter III, Section 2, Proposition 9]. Moreover, $\mu_{0} \in \mathcal{E}^{+}$because the energy is vaguely l.s.c. on $\mathfrak{M}^{+}$[11, (1.4.4)]. Since the Riesz kernel is perfect (cf. Section 2), $\mu^{K} \rightarrow \mu_{0}$ strongly in $\mathcal{E}^{+}$, and this $\mu_{0}$ is unique. As the vague topology on $\mathfrak{M}$ is Hausdorff, the unique vague limit point $\mu_{0}$ of the net $\left(\mu^{K}\right)_{K \in \mathfrak{C}}$ has to be its vague limit [2, Chapter I, Section 9, $\left.\mathrm{n}^{\circ} 1\right]$. This establishes 3.5 with $\mu^{A}:=\mu_{0}$.

It follows from 3.5 that $\mu^{A} \in \mathcal{E}_{A}^{\prime}$, and moreover

$$
\varrho\left(\mu, \mathcal{E}_{A}^{+}\right)=\varrho\left(\mu, \mathcal{E}_{A}^{\prime}\right) \leq\left\|\mu-\mu^{A}\right\|=\lim _{K \uparrow A}\left\|\mu-\mu^{K}\right\|=\lim _{K \uparrow A} \varrho\left(\mu, \mathcal{E}_{K}^{+}\right),
$$

the first equality being evident. On the other hand, for every $\nu \in \mathcal{E}_{A}^{+},\left.\nu\right|_{K} \rightarrow$ $\nu$ vaguely as $K \uparrow A$ (see e.g. [9, Lemma 1.2.2]), and therefore

$$
\|\nu\| \leq \lim _{K \uparrow A}\left\|\left.\nu\right|_{K}\right\|, \quad(\lambda, \nu) \leq \lim _{K \uparrow A}\left(\lambda,\left.\nu\right|_{K}\right) \quad \text { for every } \lambda \in \mathcal{E}^{+} .
$$

The opposite inequalities being obvious, equality in fact prevails in these inequalities; hence,

$$
\|\mu-\nu\|=\lim _{K \uparrow A}\left\|\mu-\left.\nu\right|_{K}\right\| \geq \lim _{K \uparrow A} \varrho\left(\mu, \mathcal{E}_{K}^{+}\right) \quad \text { for every } \nu \in \mathcal{E}_{A}^{+},
$$

and consequently

$$
\varrho\left(\mu, \mathcal{E}_{A}^{+}\right) \geq \lim _{K \uparrow A} \varrho\left(\mu, \mathcal{E}_{K}^{+}\right) .
$$

Combining this with 3.7 establishes 3.6.

COROLlary 3.5. Both (3.1) and 3.2 remain valid for $\mu^{A}$ defined in Theorem 3.4. Furthermore, $U^{\mu^{K}}$ increases to $U^{\mu^{A}}$ pointwise on $\mathbb{R}^{n}$ as $K \uparrow A$.

Proof. For any $K, K^{\prime} \in \mathfrak{C}_{A}$ such that $K \subset K^{\prime}$, we see from 3.2 and (3.4) that

$$
U^{\mu^{K}}=U^{\left(\mu^{K^{\prime}}\right)^{K}} \leq U^{\mu^{K^{\prime}}} \leq U^{\mu} \quad \text { on } \mathbb{R}^{n} .
$$

By Theorem 2.1, there is therefore $\nu \in \mathfrak{M}^{+}$such that $U^{\mu^{K}} \uparrow U^{\nu}$ pointwise on $\mathbb{R}^{n}$ and $\mu^{K} \rightarrow \nu$ vaguely (as $K \uparrow A$ ). This together with (3.5) yields $\nu=\mu^{A}$, the vague topology on $\mathfrak{M}$ being Hausdorff, thereby establishing the latter assertion of the corollary. Letting $K \uparrow A$ in the last display now gives 3.2 . .

Being the orthogonal projection of $\mu$ onto $\mathcal{E}_{A}^{\prime}, \mu^{A}$ is characterized by 2.3 and 2.4 with $\mathcal{M}:=\mathcal{E}_{A}^{\prime}$. Writing 2.3) for every $\nu \in \mathcal{E}_{A}^{\prime}$, and then comparing with 3.2), we obtain $\left(\mu-\mu^{A}, \nu\right)=0$, or equivalently

$$
U^{\mu^{A}}=U^{\mu} \quad \nu \text {-a.e. for every } \nu \in \mathcal{E}_{A}^{\prime} \text {. }
$$

This leads to 3.1 in a way similar to that in the proof of Theorem 3.1. Indeed, for every $K \in \mathfrak{C}_{A}$ and every $\lambda \in \mathcal{E}^{+}$, consider $\left.\lambda\right|_{E}$, where $E$ consists of all $x \in K$ with $U^{\mu^{A}}(x)<U^{\mu}(x)$. Since $\left.\lambda\right|_{E} \in \mathcal{E}_{A}^{+}$, we have $\left.\lambda\right|_{E}=0$ by $(3.8)$, and hence $c_{\alpha}(E)=0$ according to Lemma 2.2 . 
Remark 3.6. For $A$ Borel, Theorem 3.4 and Corollary 3.5 remain valid if $\mathfrak{C}_{A}$ is replaced by an increasing sequence $\left(A_{k}\right)_{k \in \mathbb{N}}$ of Borel sets whose union equals $A$. This can be seen much as above, the only delicate point being in proving the vague convergence of $\left(\left.\nu\right|_{A_{k}}\right)_{k \in \mathbb{N}}$ to $\nu \in \mathfrak{M}_{A}^{+}$. This convergence is established by applying [3, Chapter IV, Section 1, Theorem 3] to $\left(1_{A_{k}} f\right)_{k \in \mathbb{N}}$, where $f \in C_{0}\left(\mathbb{R}^{n}\right)$ is positive and $1_{Q}$ denotes the indicator function of a set $Q$.

COROllary 3.7. For $\mu \in \mathcal{E}^{+}$and $A$ arbitrary,

$$
\left(\mu, \lambda^{A}\right)=\left(\mu^{A}, \lambda\right) \quad \text { for all } \lambda \in \mathcal{E}^{+} .
$$

Identity (3.9) determines $\mu^{A}$ uniquely; that is, if it holds for $\nu \in \mathfrak{M}^{+}$in place of $\mu^{A}$, then $\nu=\mu^{A}$.

Proof. Since $\lambda^{A}, \mu^{A} \in \mathcal{E}_{A}^{\prime}$ for any $\lambda, \mu \in \mathcal{E}^{+}$, we deduce from 3.8 that

$$
\left(\mu-\mu^{A}, \lambda^{A}\right)=0 \quad \text { and } \quad\left(\lambda-\lambda^{A}, \mu^{A}\right)=0,
$$

and 3.9 follows by subtraction. If 3.9 also holds for $\nu \in \mathfrak{M}^{+}$in place of $\mu^{A}$, then

$$
U^{\mu^{A}} * m^{(r)}=U^{\nu} * m^{(r)} \quad \text { for any } r>0,
$$

where $m^{(r)}$ is the measure obtained by uniformly distributing unit mass over $B(0, r)$ and $*$ denotes convolution. Letting $r \rightarrow 0$ and applying [11, Theorem 1.11] gives $\nu=\mu^{A}$ as claimed.

Corollary 3.8. For A arbitrary,

$$
\mu^{A}=\mu \quad \text { for every } \mu \in \mathcal{E}_{A}^{\prime}\left({ }^{4}\right) \text {. }
$$

Proof. This is obvious in view of 3.6 .

3.3. Step 3: $\mu \in \mathfrak{M}^{+}$and $A$ arbitrary. Assume for a moment that $\mu$ still has finite energy. In view of $(3.5)$, we call the measure $\mu^{A}$ defined in Theorem 3.4 the inner Riesz balayage of $\mu$ to $A$. Since this $\mu^{A}$ is determined uniquely by the symmetry identity (3.9), we are thus led to the following definition of inner Riesz balayage of $\mu \in \mathfrak{M}^{+}$to $A$ arbitrary (cf. [6, p. 257]).

Definition 3.9. For $\mu \in \mathfrak{M}^{+}$and $A$ arbitrary, we call $\mu^{A} \in \mathfrak{M}^{+}$an inner balayage of $\mu$ to $A$ if

$$
E\left(\mu, \lambda^{A}\right)=E\left(\mu^{A}, \lambda\right) \quad \text { for all } \lambda \in \mathcal{E}^{+} .
$$

$\left({ }^{4}\right)$ In particular, $\mu^{A}=\mu$ for every $\mu \in \mathcal{E}_{A}^{+}$. However, this is no longer valid (not even for $A$ closed) if we drop the requirement $E(\mu)<\infty$. For instance, $\mu^{A}=\mu$ does not hold for $\mu=\varepsilon_{y}$ when $y$ is an inner $\alpha$-irregular point for $A$ (see Section 6 below). 
TheOREM 3.10. For $\mu \in \mathfrak{M}^{+}$and A arbitrary, there exists a unique inner balayage $\mu^{A} \in \mathfrak{M}^{+}$, and it satisfies both (3.1) and (3.2).

Proof. Similarly to [11, p. 272] (see also [6, p. 257, footnote]), for $\mu \in \mathfrak{M}^{+}$ one can construct a sequence $\left(\mu_{k}\right)_{k \in \mathbb{N}} \subset \mathcal{E}^{+}$such that

$$
U^{\mu_{k}} \uparrow U^{\mu} \quad \text { pointwise on } \mathbb{R}^{n} \text { (as } k \rightarrow \infty \text { ). }
$$

According to (3.8) applied to each of those $\mu_{k}$,

$$
U^{\mu_{k}^{A}}=U^{\mu_{k}} \leq U^{\mu_{k+1}}=U^{\mu_{k+1}^{A}} \quad \nu \text {-a.e. for every } \nu \in \mathcal{E}_{A}^{\prime} .
$$

In particular, $U_{k}^{\mu_{k}^{A}} \leq U^{\mu_{k+1}^{A}} \mu_{k}^{A}$-a.e., which implies by the domination principle

$$
U^{\mu_{k}^{A}} \leq U^{\mu_{k+1}^{A}} \leq U^{\mu_{k+1}} \leq U^{\mu} \quad \text { on } \mathbb{R}^{n},
$$

the second inequality being valid by $(3.2)$ (cf. Corollary 3.5).

Thus, $U^{\mu_{k}^{A}}$ increases along with $U^{\mu_{k}}($ as $k \rightarrow \infty)$ and does not exceed $U^{\mu}$. According to Theorem 2.1, there exists $\nu \in \mathfrak{M}^{+}$such that $\mu_{k}^{A} \rightarrow \nu$ vaguely and

$$
\left.U^{\mu_{k}^{A}} \uparrow U^{\nu} \quad \text { pointwise on } \mathbb{R}^{n} \text { (as } k \rightarrow \infty\right) .
$$

Writing now (3.9) for every $\mu_{k} \in \mathcal{E}^{+}, k \in \mathbb{N}$, and then applying [3, Chapter IV, Section 1, Theorem 1], which is possible in view of (3.11) and (3.13), we get (3.10) with $\mu^{A}:=\nu$. The measure $\mu^{A} \in \mathfrak{M}^{+}$is thus the required inner balayage of $\mu \in \mathfrak{M}^{+}$to $A$, and its uniqueness follows from 3.10 in the same manner as in Corollary 3.7

Relation 3.2 is obtained directly from 3.12 and 3.13 . To prove (3.1), we observe from 3.11 and 3.13 that $E:=\left\{x \in \mathbb{R}^{n}: U^{\mu^{A}}(x)<U^{\mu}(x)\right\}$ is contained in the union of the (Borel) sets $E_{k}:=\left\{x \in \mathbb{R}^{n}: U^{\mu_{k}^{A}}(x)<U^{\mu_{k}}(x)\right\}$, $k \in \mathbb{N}$. Since $c_{\alpha}\left(A \cap E_{k}\right)=0$ according to (3.1) applied to $\mu_{k}$ (cf. Corollary 3.5, Lemma 2.3 shows that, indeed, $c_{\alpha}(A \cap E)=0$.

REMARK 3.11. For any sequence (net) $\left(\mu_{k}\right) \subset \mathcal{E}^{+}$such that 3.11 holds, we thus have

$$
\mu_{k}^{A} \rightarrow \mu^{A} \quad \text { vaguely, } \quad U^{\mu_{k}^{A}} \uparrow U^{\mu^{A}} \text { pointwise on } \mathbb{R}^{n},
$$

and either of these relations may be thought of as an alternative equivalent definition of the inner swept measure $\mu^{A}$.

REMARK 3.12. Relation (3.1) no longer characterizes $\mu^{A}$ uniquely (as it does for $A$ closed and $\mu \in \mathcal{E}^{+}$, cf. Theorem 3.1, which is seen for $\mu=\varepsilon_{y}$, where $y$ is an inner $\alpha$-irregular point for $A$ (see Section 6). The uniqueness nevertheless does hold whenever $A$ is closed and $\mu \in \mathfrak{M}^{+}$is carried by $A^{c}$ (Corollary 8.4). 
REMARK 3.13. We show in Theorem 4.5 below that $\mu^{K} \rightarrow \mu^{A}$ vaguely as $K \uparrow A$, thereby justifying the term 'inner balayage'.

REMARK 3.14. In general, $\mu^{A}$ is not carried by $A$, and this is the case even for the Newtonian kernel and an open ball. What is clear so far is that $\mu^{A}$ is carried by $\bar{A}$. This will be specified in Theorem 8.5 below, providing a description of $S\left(\mu^{A}\right)$ for $A$ closed and $\mu \in \mathfrak{M}_{A^{c}}^{+}$. Also note that for any $a_{1}, a_{2} \in \mathbb{R}_{+}^{1}$ and any $\mu_{1}, \mu_{2} \in \mathfrak{M}^{+}$,

$$
\left(a_{1} \mu_{1}+a_{2} \mu_{2}\right)^{A}=a_{1} \mu_{1}^{A}+a_{2} \mu_{2}^{A} .
$$

4. Further properties of inner balayage. The following assertion shows that identity (3.10) in Definition 3.9 remains valid for $\theta \in \mathfrak{M}^{+}$in place of $\lambda \in \mathcal{E}^{+}$.

Corollary 4.1. For $\mu \in \mathfrak{M}^{+}$and A arbitrary,

$$
E\left(\mu^{A}, \theta\right)=E\left(\mu, \theta^{A}\right) \quad \text { for all } \theta \in \mathfrak{M}^{+} .
$$

Proof. For $\theta \in \mathfrak{M}^{+}$, choose $\left(\theta_{k}\right)_{k \in \mathbb{N}} \subset \mathcal{E}^{+}$so that $U^{\theta_{k}} \uparrow U^{\theta}$ pointwise on $\mathbb{R}^{n}$. Then $U_{k}^{\theta_{k}^{A}} \uparrow U^{\theta^{A}}$ pointwise on $\mathbb{R}^{n}$ (cf. Remark 3.11). But, according to 3.10 with $\lambda=\theta_{k}$,

$$
E\left(\mu^{A}, \theta_{k}\right)=E\left(\mu, \theta_{k}^{A}\right) .
$$

Letting here $k \rightarrow \infty$, we obtain (4.1) by [3, Chapter IV, Section 1 , Theorem 1].

Corollary 4.2. For any $\mu \in \mathfrak{M}^{+}$and any $Q \subset A$,

$$
\mu^{Q}=\left(\mu^{A}\right)^{Q} .
$$

Proof. For every $\lambda \in \mathcal{E}^{+}$, from 3.10) we obtain

$$
E\left(\left(\mu^{A}\right)^{Q}, \lambda\right)=E\left(\mu^{A}, \lambda^{Q}\right)=E\left(\mu,\left(\lambda^{Q}\right)^{A}\right)=E\left(\mu, \lambda^{Q}\right)=E\left(\mu^{Q}, \lambda\right),
$$

the equality $\left(\lambda^{Q}\right)^{A}=\lambda^{Q}$ being valid by Corollary 3.8 applied to $\lambda^{Q} \in \mathcal{E}_{Q}^{\prime}$ $\subset \mathcal{E}_{A}^{\prime}$. Taking here $\lambda=m^{(r)}$ and letting $r \downarrow 0$ gives 4.2 (cf. the proof of Corollary 3.7.

TheOREM 4.3 (Characteristic property). For $\mu \in \mathfrak{M}^{+}$and A arbitrary, $U^{\mu^{A}}$ can be characterized uniquely by the extremal property

$$
U^{\mu^{A}}=\min _{\xi \in \Xi_{A}} U^{\xi}
$$

where $\Xi_{A}$ consists of all $\xi \in \mathfrak{M}^{+}$with

$$
U^{\xi} \geq U^{\mu} \quad \text { n.e. on } A \text {. }
$$

Proof. Since $\mu^{A} \in \Xi_{A}$ by (3.1) (cf. Theorem 3.10), it is enough to establish

$$
U^{\mu^{A}} \leq U^{\xi} \quad \text { on } \mathbb{R}^{n},
$$


where $\xi \in \Xi_{A}$ is fixed. As $U^{\mu^{A}}$ is the pointwise limit of an increasing sequence $\left(U^{\mu_{k}^{A}}\right)_{k \in \mathbb{N}}$ with $\left(\mu_{k}\right)_{k \in \mathbb{N}} \subset \mathcal{E}^{+}$suitably chosen (cf. Remark 3.11), it suffices to verify (4.4) for $\mu \in \mathcal{E}^{+}$. By 3.2 applied to $K \in \mathfrak{C}_{A}$, we have $U^{\mu^{K}} \leq U^{\mu}$ on $\mathbb{R}^{n}$, which together with 4.3 shows that the inequality

$$
U^{\mu^{K}} \leq U^{\xi}
$$

holds n.e. on $K$, hence $\mu^{K}$-a.e. because $\mu^{K} \in \mathcal{E}_{K}^{+}$, and consequently on all of $\mathbb{R}^{n}$ by the domination principle. On account of Corollary 3.5, letting here $K \uparrow A$ results in (4.4) as required.

Corollary 4.4. For $\mu \in \mathfrak{M}^{+}$and A arbitrary,

$$
\mu^{A}=\mu^{A^{\prime}}
$$

whenever $\left(A \backslash A^{\prime}\right) \cup\left(A^{\prime} \backslash A\right)$ is of inner capacity zero.

Proof. Indeed, $U^{\mu^{A^{\prime}}}=U^{\mu}$ n.e. on $A^{\prime}$, hence n.e. on $A$. Therefore, by Theorem 4.3. $U^{\mu^{A}} \leq U^{\mu^{A^{\prime}}}$ on $\mathbb{R}^{n}$. As the same holds with $A$ and $A^{\prime}$ interchanged, (4.5) follows.

TheOrEm 4.5. For $\mu \in \mathfrak{M}^{+}$and $A$ arbitrary, $U^{\mu^{K}} \uparrow U^{\mu^{A}}$ pointwise on $\mathbb{R}^{n}$ and $\mu^{K} \rightarrow \mu^{A}$ vaguely as $K \uparrow A$. If moreover $E(\mu)<\infty$, then $\mu^{K} \rightarrow \mu^{A}$ also strongly.

Proof. For $\mu \in \mathcal{E}^{+}$, this has already been established in Theorem 3.1 and Corollary 3.5. It thus remains to prove the former assertion for $\mu \in \mathfrak{M}^{+} \backslash \mathcal{E}^{+}$.

According to 4.2), for any $K, K^{\prime} \in \mathfrak{C}_{A}$ such that $K \subset K^{\prime}$ we have $\mu^{K}=\left(\mu^{K^{\prime}}\right)^{K}$. In view of 3.2 , this implies that the net $\left(U^{\mu^{K}}\right)_{K \in \mathfrak{C}_{A}}$ is increasing and majorized by $U^{\mu}$. By Theorem 2.1, there is therefore $\nu \in \mathfrak{M}^{+}$ such that $U^{\mu^{K}} \uparrow U^{\nu}$ pointwise on $\mathbb{R}^{n}$ and $\mu^{K} \rightarrow \nu$ vaguely as $K \uparrow A$. The proof is completed by showing that $\nu=\mu^{A}$, or equivalently

$$
E(\nu, \lambda)=E\left(\mu, \lambda^{A}\right)
$$

for any given $\lambda \in \mathcal{E}^{+}$(cf. Definition 3.9). Indeed, according to 3.10 applied to $K$,

$$
E\left(\mu^{K}, \lambda\right)=E\left(\mu, \lambda^{K}\right) \quad \text { for every } K \in \mathfrak{C}_{A},
$$

while $U^{\lambda^{A}}$ is the pointwise limit of the increasing net $\left(U^{\lambda^{K}}\right)_{K \in \mathfrak{C}_{A}}$ (see Corollary 3.5]. Letting $K \uparrow A$, we obtain the required identity by [3], Chapter IV, Section 1, Theorem 1].

Corollary 4.6. Fix $\mu \in \mathcal{E}^{+}$and $A \subset \mathbb{R}^{n}$. For every $\varepsilon>0$, there exists $K_{0} \in \mathfrak{C}_{A}$ with the property $\left\|\mu^{A}-\mu^{K}\right\|<\varepsilon$ for all $K \in \mathfrak{C}_{A}$ such that $K \supset K_{0}$.

Theorem 4.7. For A Borel, Theorem 4.5 and Corollary 4.6 remain valid if $\mathfrak{C}_{A}$ is replaced by an increasing sequence $\left(A_{k}\right)_{k \in \mathbb{N}}$ of Borel sets with union $A$. 
Proof. Since for every $\lambda \in \mathcal{E}^{+}, U^{\lambda^{A_{k}} \uparrow} U^{\lambda^{A}}$ pointwise on $\mathbb{R}^{n}$ as $k \rightarrow \infty$ (see Remark 3.6), the proof runs as above.

The following result can certainly be extended to a general perfect kernel on a locally compact space, which is, however, outside the scope of this study.

THEOREM 4.8. Assume $A$ is the intersection of a lower directed family $\left(A_{t}\right)_{t \in T}$ of closed sets. For any $\mu \in \mathcal{E}^{+}, \mu^{A_{t}} \rightarrow \mu^{A}$ strongly and vaguely.

Proof. For $\mu \in \mathcal{E}^{+}, \mu^{A_{t}}$ is the orthogonal projection of $\mu$ onto $\mathcal{E}_{A_{t}}^{+}$ (cf. Theorem 3.1. Since $\mathcal{E}_{A}^{+} \subset \mathcal{E}_{A_{t^{\prime}}}^{+} \subset \mathcal{E}_{A_{t}}^{+}$whenever $t^{\prime}$ follows $t$, the net $\left(\varrho\left(\mu, \mathcal{E}_{A_{t}}^{+}\right)\right)_{t \in T}$ is increasing and majorized by $\varrho\left(\mu, \mathcal{E}_{A}^{+}\right)<\infty$; hence, it is Cauchy in $\mathbb{R}$. In consequence of [9, Lemma 4.1.1] with $\mathcal{H}:=\mathcal{E}, \Gamma:=\{\mu-\nu$ : $\left.\nu \in \mathcal{E}_{A_{t}}^{+}\right\}$, and $\lambda:=\mu-\mu^{A_{t}}$,

$$
\left\|\mu^{A_{t}}-\mu^{A_{t^{\prime}}}\right\|^{2}=\left\|\left(\mu-\mu^{A_{t}}\right)-\left(\mu-\mu^{A_{t^{\prime}}}\right)\right\|^{2} \leq \varrho\left(\mu, \mathcal{E}_{A_{t^{\prime}}}^{+}\right)^{2}-\varrho\left(\mu, \mathcal{E}_{A_{t}}^{+}\right)^{2} .
$$

It follows that the net $\left(\mu^{A_{t}}\right)_{t \in T}$ is strong Cauchy in $\mathcal{E}^{+}$. Being thus strongly bounded, it is vaguely bounded by [9, Lemma 2.5.1], and therefore has a vague limit point $\mu_{0} \in \mathfrak{M}^{+}$according to [3. Chapter III, Section 2, Proposition 9]. On account of the perfectness of the Riesz kernel, this $\mu_{0}$ is in fact the unique strong and vague limit of $\left(\mu^{A_{t}}\right)_{t \in T}$. Consequently, $\mu_{0}$ belongs to $\mathcal{E}_{A_{t}}^{+}$ for every $t \in T$, and hence to $\mathcal{E}_{A}^{+}$, the intersection of $\mathcal{E}_{A_{t}}^{+}$over $t \in T$. Thus,

$$
\varrho\left(\mu, \mathcal{E}_{A}^{+}\right) \leq\left\|\mu-\mu_{0}\right\|=\lim _{t}\left\|\mu-\mu^{A_{t}}\right\|=\lim _{t} \varrho\left(\mu, \mathcal{E}_{A_{t}}^{+}\right) \leq \varrho\left(\mu, \mathcal{E}_{A}^{+}\right)
$$

which implies $\mu_{0}=\mu^{A}$.

The following corollary to Theorem 4.5 will be specified in Theorems 8.6 and 8.7 below.

Corollary 4.9. For $\mu \in \mathfrak{M}^{+}$and $A$ arbitrary,

$$
\mu^{A}\left(\mathbb{R}^{n}\right) \leq \mu\left(\mathbb{R}^{n}\right)
$$

Proof. Since $\mu^{K} \rightarrow \mu^{A}$ vaguely as $K \uparrow A$, while the map $\nu \mapsto \nu\left(\mathbb{R}^{n}\right)$ is vaguely l.s.c. on $\mathfrak{M}^{+}$, it suffices to establish 4.6 for $A=K$ compact. Consider a closed ball $\bar{B}$ containing $K$, and the equilibrium measure $\gamma$ on $\bar{B}$; then $U^{\gamma}=1$ on $\bar{B}$ and $U^{\gamma} \leq 1$ on $\mathbb{R}^{n}$ [11, Chapter II, Section 3, $\left.\mathrm{n}^{\circ} 13\right]$. Therefore,

$$
\mu^{K}\left(\mathbb{R}^{n}\right)=\int U^{\gamma} d \mu^{K}=\int U^{\mu^{K}} d \gamma \leq \int U^{\mu} d \gamma=\int U^{\gamma} d \mu \leq \mu\left(\mathbb{R}^{n}\right),
$$

the former inequality being valid according to 3.2 (cf. Theorem 3.10).

5. Inner Riesz equilibrium measure. Criteria for its existence. We assume as above that $A$ is an arbitrary proper subset of $\mathbb{R}^{n}$ with $c_{\alpha}(A)$ $>0$, and denote by $\Theta_{A}$ the class of all $\nu \in \mathfrak{M}^{+}$with $U^{\nu} \geq 1$ n.e. on $A$. 
Definition 5.1. $\gamma_{A} \in \mathfrak{M}^{+}$is said to be an inner equilibrium measure of $A$ if (its potential $U^{\gamma_{A}}$ is not identically infinite and) $\left(^{5}\right)$

$$
U^{\gamma_{A}}=\inf _{\nu \in \Theta_{A}} U^{\nu}
$$

An inner equilibrium measure $\gamma_{A}$ is certainly unique, and it exists only if $\Theta_{A}$ is nonempty. (We shall show in Lemma 5.3 below that the latter can actually be reversed, and so $\Theta_{A} \neq \emptyset$ is necessary and sufficient for the existence of $\gamma_{A}$.)

Lemma 5.2. Assume that $c_{\alpha}(A)<\infty$. Then $\gamma_{A}$ exists, and moreover

(a) $\gamma_{A}\left(\mathbb{R}^{n}\right)=E\left(\gamma_{A}\right)=c_{\alpha}(A)$,

(b) $S\left(\gamma_{A}\right) \subset \bar{A}$,

(c) $U^{\gamma_{A}}=1$ n.e. on $A$,

(d) $U^{\gamma_{A}} \leq 1$ on $\mathbb{R}^{n}$.

This $\gamma_{A}$ is the unique solution to the problem of minimizing the energy over $\Theta_{A} \cap \mathcal{E}^{+}$, and hence it is characterized uniquely within $\mathcal{E}^{+}$by (a) and (c).

Proof. This is obtained from [11, Chapter II, Section 2, $\mathrm{n}^{\circ}$ 7] and [11, Lemma 4.5]. See also [9, Section 4.1].

Lemma 5.3. For $A$ arbitrary, assume that $\Theta_{A} \neq \emptyset$. Then $\gamma_{A}$ exists. Furthermore, it is $c_{\alpha}$-absolutely continuous and has the properties $S\left(\gamma_{A}\right) \subset \bar{A}$ and $\left({ }^{6}\right)$

$$
\begin{array}{ll}
U^{\gamma_{A}}=1 & \text { n.e. on } A, \\
U^{\gamma_{A}} \leq 1 & \text { on } \mathbb{R}^{n}, \\
\gamma_{K} \rightarrow \gamma_{A} & \text { vaguely as } K \uparrow A, \\
U^{\gamma_{K}} \uparrow U^{\gamma_{A}} & \text { pointwise on } \mathbb{R}^{n} \text { as } K \uparrow A .
\end{array}
$$

Proof. Fix $\xi \in \mathfrak{M}^{+}$with $U^{\xi} \geq 1$ n.e. on $A$. Then for any $K, K^{\prime} \in \mathfrak{C}_{A}$ such that $K \subset K^{\prime}$, we have $U^{\gamma_{K}}=U^{\gamma_{K^{\prime}}} \leq U^{\xi}$ n.e. on $K$ (cf. Lemma 5.2(c)), and hence $\gamma_{K}$-a.e. The domination principle therefore shows that the net $\left(U^{\gamma_{K}}\right)_{K \in \mathfrak{C}_{A}}$ is pointwise increasing on $\mathbb{R}^{n}$ and majorized by $U^{\xi}$. According to Theorem 2.1. there exists $\nu_{0} \in \mathfrak{M}^{+}$such that $U^{\gamma_{K}} \uparrow U^{\nu_{0}}$ pointwise on $\mathbb{R}^{n}$ and $\gamma_{K} \rightarrow \nu_{0}$ vaguely as $K \uparrow A$. Hence, $U^{\nu_{0}} \leq 1$ on $\mathbb{R}^{n}$ because $U^{\gamma_{K}} \leq 1$ on $\mathbb{R}^{n}$ by Lemma 5.2(d).

We claim that $U^{\nu_{0}}=1$ n.e. on $A$, or equivalently n.e. on every $K \in \mathfrak{C}_{A}$. We thus need to prove $c_{\alpha}(E)=0$, where $E:=\left\{x \in K: U^{\nu_{0}}(x)<1\right\}$. But

$\left({ }^{5}\right)$ One can introduce a concept of inner balayage for positive $\alpha$-superharmonic functions on $\mathbb{R}^{n}$, generalizing that by Cartan for $\alpha=2$ [6. p. 257], and then $U^{\gamma_{A}}$ (if $\gamma_{A}$ exists) will be thought of as an inner balayage of $f \equiv 1$ to $A$. Being however mainly concerned with the existence of $\gamma_{A}$, we drop this part of the analysis.

$\left({ }^{6}\right)$ Relation 5.1 will be specified below (cf. Lemma 6.11 and Theorem 6.6. See also Theorem 7.2 which establishes a detailed description of $U^{\gamma_{A}}$ and $S\left(\gamma_{A}\right)$ for $A$ closed. 
this is obvious in view of the relations $E \subset E^{\prime}:=\left\{x \in K: U^{\gamma_{K}}(x)<1\right\}$ and $c_{\alpha}\left(E^{\prime}\right)=0$, the latter being seen from Lemma 5.2. (c).

Thus, $\nu_{0} \in \Theta_{A}$. We assert that this $\nu_{0}$ actually serves as an inner equilibrium measure of $A$. According to Definition 5.1, it is enough to verify that $U^{\nu_{0}} \leq U^{\nu}$ on $\mathbb{R}^{n}$ for every $\nu \in \mathfrak{M}^{+}$with $U^{\nu} \geq 1$ n.e. on $A$. Since then $U^{\gamma_{K}} \leq U^{\nu}$ on $\mathbb{R}^{n}$ for every $K \in \mathfrak{C}_{A}$ (see above), the required inequality is obtained by letting $K \uparrow A$.

It has thus been proven that $\gamma_{A}:=\nu_{0}$ exists and satisfies (5.1)-(5.4). Next, it follows from 5.3 that $S\left(\gamma_{A}\right) \subset \bar{A}, \mathfrak{M}^{+}(\bar{A})$ being vaguely closed. Finally, since the restriction of $\gamma_{A}$ to any compact subset of $\mathbb{R}^{n}$ is of finite energy because of (5.2), $\gamma_{A}$ is $c_{\alpha}$-absolutely continuous.

Corollary 5.4. For A closed, assume that there is an $c_{\alpha}$-absolutely continuous measure $\check{\gamma} \in \mathfrak{M}_{A}^{+}$with $U^{\check{\gamma}}=1$ n.e. on $A$. Then $\gamma_{A}$ exists, and moreover $\gamma_{A}=\check{\gamma}$.

Proof. Since $\check{\gamma} \in \Theta_{A}, \gamma_{A}$ exists according to the preceding lemma. Furthermore, $\gamma_{A}$ is $c_{\alpha}$-absolutely continuous, supported by $A$, and satisfies (5.1). Then necessarily $\gamma_{A}=\check{\gamma}$, because any two $c_{\alpha}$-absolutely continuous measures of the class $\mathfrak{M}_{A}^{+}$coincide whenever their potentials are equal n.e. on $A$ (cf. [11, p. 178, Remark]).

In the case where $A$ is Borel, Theorem 5.5 below is [11, Theorem 5.1]. However, [11, Theorem 5.1] has not been completely justified, because the proof of its necessity part is based on the concept of balayage, introduced in [11, Chapter IV, Section 6, $\mathrm{n}^{\circ} 25$ ] by means of the integral representation (1.1) (see the Introduction for details). Applying the theory of inner Riesz balayage, developed in Section 3 above, we fix the gap in [11, proof of Theorem 5.1], and moreover we strengthen [11, Theorem 5.1] to $A$ arbitrary.

TheOrem 5.5. For A arbitrary, the following two assertions are equivalent.

(i) There exists an inner equilibrium measure $\gamma_{A}$.

(ii) If $R_{k}:=\left\{x \in \mathbb{R}^{n}: q^{k} \leq|x|<q^{k+1}\right\}$, where $q \in(1, \infty)$, then

$$
\sum_{k \in \mathbb{N}} \frac{c_{\alpha}\left(A \cap R_{k}\right)}{q^{k(n-\alpha)}}<\infty .
$$

Proof. Assuming first that (ii) holds, write $\gamma_{k}:=\gamma_{A_{k}}$, where $A_{k}:=$ $A \cap R_{k}$; this $\gamma_{A_{k}}$ exists according to Lemma 5.2. It is seen from Lemma 5.3 that (i) will follow once we establish

$$
\xi:=\sum_{k \in \mathbb{N}} \gamma_{k} \in \Theta_{A}
$$


To this end, we first observe that $U^{\xi} \not \equiv \infty$, or equivalently (cf. 2.1)

$$
I:=\int_{|x| \geq q} \frac{d \xi(x)}{|x|^{n-\alpha}}<\infty .
$$

Indeed,

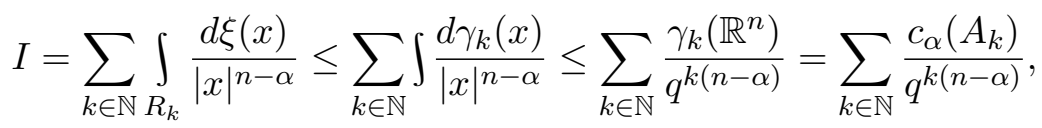

the last equality being valid by Lemma 5.2(a); therefore, $I<\infty$ by (5.5). The proof of 5.6 is thus reduced to establishing $U^{\xi} \geq 1$ n.e. on $A$. Since the sets $R_{k}, k \in \mathbb{N}$, are Borel, this follows from $U^{\xi} \geq U^{\gamma_{k}}=1$ n.e. on $A \cap R_{k}$ by applying Lemma 2.3 .

Assuming now that $\gamma_{A}$ exists, we complete the proof by showing

$$
S_{1}:=\sum_{k \in \mathbb{N}} \frac{c_{\alpha}\left(A_{2 k}\right)}{q^{2 k(n-\alpha)}}<\infty, \quad S_{2}:=\sum_{k \in \mathbb{N}} \frac{c_{\alpha}\left(A_{2 k-1}\right)}{q^{(2 k-1)(n-\alpha)}}<\infty
$$

(cf. (5.5)). Since both these series can be handled in the same manner, we shall establish $S_{1}<\infty$. Write

$$
A^{\prime}:=\bigcup_{k \in \mathbb{N}} A_{2 k},
$$

$\gamma^{\prime}:=\left.\gamma_{A}\right|_{\overline{A^{\prime}}}, \gamma^{\prime \prime}:=\gamma_{A}-\gamma^{\prime}$, and

$$
\tilde{\gamma}:=\gamma^{\prime}+\left(\gamma^{\prime \prime}\right)^{A^{\prime}}
$$

where the existence of the inner balayage $\left(\gamma^{\prime \prime}\right)^{A^{\prime}}$ (cf. Definition 3.9 is justified by Theorem 3.10 (compare with [11, proof of Theorem 5.1]). According to

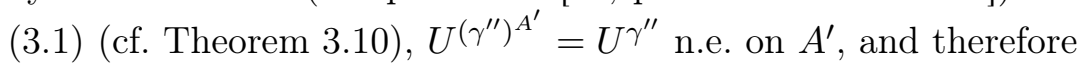

$$
U^{\tilde{\gamma}}=U^{\gamma^{\prime}+\left(\gamma^{\prime \prime}\right)^{A^{\prime}}}=U^{\gamma^{\prime}+\gamma^{\prime \prime}}=U^{\gamma_{A}}=1 \quad \text { n.e. on } A^{\prime} .
$$

Noting that

$$
\sum_{k \in \mathbb{N}} \frac{\tilde{\gamma}\left(\overline{A_{2 k}}\right)}{q^{(2 k+1)(n-\alpha)}} \leq \sum_{k \in \mathbb{N}} \int_{R_{2 k}} \frac{d \tilde{\gamma}(x)}{|x|^{n-\alpha}} \leq \int_{|x|>1} \frac{d \tilde{\gamma}(x)}{|x|^{n-\alpha}}<\infty,
$$

the last inequality being valid by $(2.1)$, we obtain

$$
\sum_{k \in \mathbb{N}} \frac{\tilde{\gamma}\left(\overline{A_{2 k}}\right)}{q^{2 k(n-\alpha)}}<\infty .
$$

Hence, $S_{1}<\infty$ will follow if we show

$$
c_{\alpha}\left(A_{2 k}\right) \leq M \tilde{\gamma}\left(\overline{A_{2 k}}\right) \quad \text { for all } k \in \mathbb{N},
$$

where $M \in(0, \infty)$ is independent of $k$. The proof of this is based on 5.7) and runs in the same manner as in [11, pp. 282-283]. 
REMARK 5.6. The finiteness of $c_{\alpha}(A)$ is sufficient for the existence of an inner equilibrium measure $\gamma_{A}$ (cf. Lemma 5.2), but not necessary. This can be seen by comparing Theorem 5.5 with the following assertion. if

Lemma 5.7 (see [11, Lemma 5.5]). For A Borel, $c_{\alpha}(A)<\infty$ if and only

$$
\sum_{k \in \mathbb{N}} \frac{c_{\alpha}\left(A \cap R_{k}\right)}{q^{2 k(n-\alpha)}}<\infty,
$$

where $R_{k}, k \in \mathbb{N}$, are defined in Theorem 5.5 and $q \in(1, \infty)$.

The next example can be obtained from Theorem 5.5 and Lemma 5.7 by analyzing estimates in [11, Chapter V, Section 1, Example].

Example 5.8. Let $n=3$ and $\alpha=2$. Define $A$ to be a rotation body

$$
A:=\left\{x \in \mathbb{R}^{3}: 0 \leq x_{1}<\infty, x_{2}^{2}+x_{3}^{2} \leq \varrho^{2}\left(x_{1}\right)\right\},
$$

where $\varrho$ is given by one of the following three formulae:

$$
\begin{array}{ll}
\varrho\left(x_{1}\right)=x_{1}^{-s} & \text { with } s \in[0, \infty), \\
\varrho\left(x_{1}\right)=\exp \left(-x_{1}^{s}\right) & \text { with } s \in(0,1], \\
\varrho\left(x_{1}\right)=\exp \left(-x_{1}^{s}\right) & \text { with } s \in(1, \infty) .
\end{array}
$$

Then $\gamma_{A}$ does not exist if $\varrho$ is defined by (5.9), $\gamma_{A}$ exists but $c_{2}(A)=\infty$ if $\varrho$ is given by (5.10) (see Figure 1), and finally $c_{2}(A)<\infty$ if (5.11) holds.

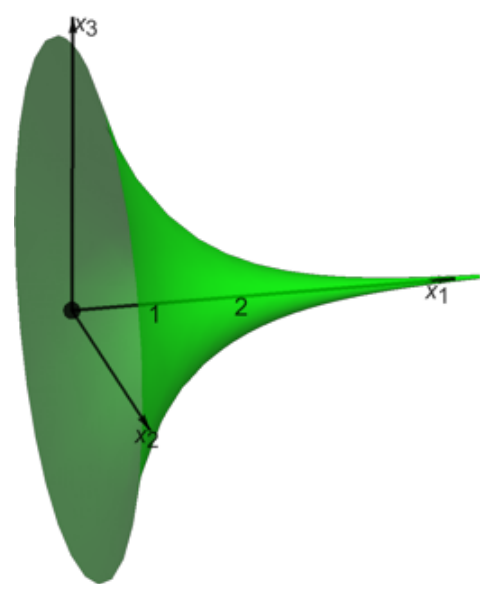

Fig. 1. $A:=\left\{0 \leq x_{1}<\infty, x_{2}^{2}+x_{3}^{2} \leq \rho^{2}\left(x_{1}\right)\right\}$ with $\rho\left(x_{1}\right)=\exp \left(-x_{1}\right)$

\section{Wiener type criterion of inner $\alpha$-irregularity}

Definition 6.1. A point $y \in \mathbb{R}^{n}$ is said to be inner $\alpha$-irregular for $A$ if $y \in \bar{A}$ and $\varepsilon_{y}^{A} \neq \varepsilon_{y}$, where $\varepsilon_{y}^{A}$ is the inner balayage of $\varepsilon_{y}$ to $A$ (cf. Definition 3.9. All other points of $\bar{A}$ are said to be inner $\alpha$-regular for $A$. 
Remark 6.2. For every $y \notin \bar{A}$, we have $\varepsilon_{y}^{A} \in \mathcal{E}^{+}$, and therefore $\varepsilon_{y}^{A} \neq \varepsilon_{y}$. Indeed, in view of (3.2) (cf. Theorem 3.10),

$$
U^{\varepsilon_{y}^{A}}(x) \leq U^{\varepsilon_{y}}(x)=|x-y|^{\alpha-n} \leq \max _{z \in \bar{A}}|z-y|^{\alpha-n}<\infty \quad \text { for all } x \in \bar{A} .
$$

Since $\varepsilon_{y}^{A}\left(\mathbb{R}^{n}\right) \leq 1$ according to $4.6, E\left(\varepsilon_{y}^{A}\right)<\infty$ follows.

Lemma 6.3. $y$ is inner $\alpha$-regular for $A$ (if and) only if

$$
U^{\mu^{A}}(y)=U^{\mu}(y) \quad \text { for every } \mu \in \mathfrak{M}^{+} .
$$

Proof. If $y$ is inner $\alpha$-regular for $A$, then 4.1) applied to $\theta=\varepsilon_{y}$ gives

$$
U^{\mu^{A}}(y)=E\left(\mu^{A}, \varepsilon_{y}\right)=E\left(\mu, \varepsilon_{y}^{A}\right)=E\left(\mu, \varepsilon_{y}\right)=U^{\mu}(y)
$$

for every $\mu \in \mathfrak{M}^{+}$, which is our claim.

Let $A_{I}$ consist of all inner $\alpha$-irregular points for $A$.

Theorem 6.4 (Wiener type criterion). $y \in A_{I}$ if and only if

$$
\sum_{k \in \mathbb{N}} \frac{c_{\alpha}\left(A_{k}\right)}{q^{k(n-\alpha)}}<\infty,
$$

where $A_{k}:=A \cap\left\{x \in \mathbb{R}^{n}: q^{k+1}<|x-y| \leq q^{k}\right\}$ and $q \in(0,1)$.

Theorem 6.4 follows directly from Lemmas 6.76 .9 below. Theorem 6.4 implies, in turn, the next two assertions (see Section 6.2 for the proof of the latter).

Corollary 6.5. $A_{I} \subset \partial A$.

Theorem 6.6. $c_{\alpha}\left(A_{I} \cap A\right)=0$.

6.1. Auxiliary assertions. For any $y \in \mathbb{R}^{n}$, define the inversion $J_{y}$ with respect to $S(y, 1)$ mapping each point $x \neq y$ to the point $x^{*}$ on the ray through $x$ issuing from $y$ which is uniquely determined by

$$
|x-y| \cdot\left|x^{*}-y\right|=1 \text {. }
$$

This is a homeomorphism of $\mathbb{R}^{n} \backslash\{y\}$ onto itself; furthermore,

$$
\left|x^{*}-z^{*}\right|=\frac{|x-z|}{|x-y||z-y|} \quad \text { for all } x, z \in \mathbb{R}^{n} \backslash\{y\} .
$$

It can be extended to a homeomorphism of the one-point compactification $\overline{\mathbb{R}^{n}}$ of $\mathbb{R}^{n}$ onto itself such that $y$ and the point at infinity are mapped to each other.

In Lemmas 6.76 .9 below, $y \in \mathbb{R}^{n}$ is fixed and $A^{*}$ the $J_{y}$-image of $A \backslash\{y\}$.

Lemma 6.7. Relation (6.2) holds if and only if an inner equilibrium measure $\gamma_{A^{*}}$ of $A^{*}$ exists. 
Proof. Let $q \in(0,1)$ and $A_{k}$ be as in Theorem 6.4. It follows from 6.3. that

$$
q^{-2 k}|x-z| \leq\left|x^{*}-z^{*}\right| \leq q^{-2 k-2}|x-z| \quad \text { for any } x, z \in A_{k},
$$

and hence, by [11, Remark to Theorem 2.9],

$$
q^{-2 k(n-\alpha)} c_{\alpha}\left(A_{k}\right) \leq c_{\alpha}\left(A_{k}^{*}\right) \leq q^{-(2 k+2)(n-\alpha)} c_{\alpha}\left(A_{k}\right),
$$

where

$$
A_{k}^{*}:=J_{y}\left(A_{k}\right)=A^{*} \cap\left\{x \in \mathbb{R}^{n}: q^{-k} \leq|x-y|<q^{-k-1}\right\} .
$$

Therefore, 6.2 holds if and only if

$$
\sum_{k \in \mathbb{N}} q^{k(n-\alpha)} c_{\alpha}\left(A_{k}^{*}\right)<\infty,
$$

which according to Theorem 5.5 is equivalent to the existence of $\gamma_{A^{*}}$.

To each $\nu \in \mathfrak{M}^{+}$with $\nu(\{y\})=0$ we assign the Kelvin transform $\nu^{*}=$ $\mathcal{K}_{y} \nu=\mathcal{K}_{y}(\nu) \in \mathfrak{M}^{+}$(see [12] or [11, Chapter IV, Section $\left.5, \mathrm{n}^{\circ} 19\right]$ ) by means of

$$
d \nu^{*}\left(x^{*}\right)=|x-y|^{\alpha-n} d \nu(x), \quad \text { where } \quad x^{*}:=J_{y}(x) \in \mathbb{R}^{n} .
$$

Then $\mathcal{K}_{y}$ is an involution, i.e. $\mathcal{K}_{y}\left(\mathcal{K}_{y} \nu\right)=\nu$, which implies in view of 6.5 that

$$
\nu\left(\mathbb{R}^{n}\right)=U^{\nu^{*}}(y) .
$$

Next, combining (6.5) and (6.3) yields

$$
U^{\nu^{*}}\left(x^{*}\right)=|x-y|^{n-\alpha} U^{\nu}(x) \quad \text { for all } x^{*} \in \mathbb{R}^{n},
$$

and therefore

$$
E\left(\mu^{*}, \nu^{*}\right)=E(\mu, \nu)
$$

for every $\mu \in \mathfrak{M}^{+}$with $\mu(\{y\})=0$. Equality 6.8 is obtained by multiplying (6.5) (with $\mu$ in place of $\nu$ ) by (6.7), and then integrating with respect to $d \mu(x)$ over $\mathbb{R}^{n}$.

Lemma 6.8. Assume $\gamma_{A^{*}}$ exists. For its Kelvin transform $\left(\gamma_{A^{*}}\right)^{*}=$ $\mathcal{K}_{y} \gamma_{A^{*}}$, we have

$$
\left(\gamma_{A^{*}}\right)^{*}=\varepsilon_{y}^{A},
$$

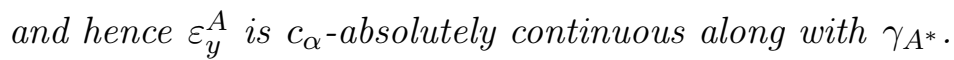

Proof. Assume that $A \not \ngtr y$, which certainly involves no loss of generality (cf. Corollary 4.4). Then $J_{y}$ is an order-preserving one-to-one mapping of $\mathfrak{C}_{A}$ onto $\mathfrak{C}_{A^{*}}$. In view of the $c_{\alpha}$-absolute continuity of inner equilibrium measure (cf. Lemma 5.3), one can consider the Kelvin transforms $\left(\gamma_{A^{*}}\right)^{*}=\mathcal{K}_{y} \gamma_{A^{*}}$ 
and $\left(\gamma_{K^{*}}\right)^{*}=\mathcal{K}_{y} \gamma_{K^{*}}$ for every $K^{*}:=J_{y}(K) \in \mathfrak{C}_{A^{*}}$. It follows from (5.4) applied to $A^{*}$, and (6.7) applied to each of $\gamma_{K^{*}}$ and $\gamma_{A^{*}}$, that

$$
\left.U^{\left(\gamma_{K^{*}}\right)^{*}} \uparrow U^{\left(\gamma_{A^{*}}\right)^{*}} \quad \text { pointwise on } \mathbb{R}^{n} \text { (as } K^{*} \uparrow A^{*}\right) \text {. }
$$

Also observe that $\left(\gamma_{K^{*}}\right)^{*} \in \mathcal{E}_{K}^{+}$, which is seen from 6.8 with $\mu=\nu=\gamma_{K^{*}}$.

We begin by establishing

$$
\left(\gamma_{K^{*}}\right)^{*}=\varepsilon_{y}^{K} \quad \text { for every } K \in \mathfrak{C}_{A} .
$$

Combining 6.7 applied to $\gamma_{K^{*}}$ with 5.1 applied to $K^{*}$ gives $\left({ }^{7}\right)$

$$
\begin{aligned}
U^{\left(\gamma_{K^{*}}\right)^{*}}(x) & =\left|x^{*}-y\right|^{n-\alpha} U^{\gamma_{K^{*}}}\left(x^{*}\right)=\left|x^{*}-y\right|^{n-\alpha} \\
& =|x-y|^{\alpha-n}=U^{\varepsilon_{y}}(x) \quad \text { n.e. on } K,
\end{aligned}
$$

which is 4.3 with $\xi=\left(\gamma_{K^{*}}\right)^{*}$ and $\mu=\varepsilon_{y}$. According to Theorem 4.3, 6.11) will follow once we verify that $U^{\xi} \geq U^{\left(\gamma_{\left.K^{*}\right)^{*}}\right.}$ on $\mathbb{R}^{n}$ for any $\xi \in \mathfrak{M}^{+}$with

$$
U^{\xi} \geq U^{\varepsilon_{y}} \quad \text { n.e. on } K \text {. }
$$

As seen from the last two displays, the inequality in question holds, indeed, n.e. on $K$, hence $\left(\gamma_{K^{*}}\right)^{*}$-a.e. because $\left(\gamma_{K^{*}}\right)^{*} \in \mathcal{E}_{K}^{+}$, and therefore, by the domination principle, on all of $\mathbb{R}^{n}$, as required.

But, according to Theorem 4.5 with $\mu=\varepsilon_{y}$,

$$
\left.U^{\varepsilon_{y}^{K}} \uparrow U^{\varepsilon_{y}^{A}} \quad \text { pointwise on } \mathbb{R}^{n} \text { (as } K \uparrow A\right) .
$$

Now substituting (6.11) into this display, and then comparing the relation thus obtained with 6.10, we get 6.9.

Lemma 6.9. If $\gamma_{A^{*}}$ does not exist, then

$$
\varepsilon_{y}^{A}=\varepsilon_{y},
$$

and hence $y$ is inner $\alpha$-regular for $A$.

Proof. Assuming $\gamma_{A^{*}}$ does not exist, we begin by observing that then $\varepsilon_{y}^{A}(\{y\})>0$. Indeed, if not, then the Kelvin transform $\left(\varepsilon_{y}^{A}\right)^{*}=\mathcal{K}_{y} \varepsilon_{y}^{A}$ exists and has the potential equal to 1 n.e. on $A^{*}$, the latter being seen by applying (3.1) and 6.7 to $\varepsilon_{y}$ and $\varepsilon_{y}^{A}$, respectively. Hence, $\left(\varepsilon_{y}^{A}\right)^{*} \in \Theta_{A^{*}}$, which by Lemma 5.3 (applied to $A^{*}$ ) contradicts our assumption.

We proceed to show that the relation $\varepsilon_{y}^{A}(\{y\})>0$ thus obtained implies $\varepsilon_{y}^{A}=\varepsilon_{y}$. Indeed, if not, then

$$
\varepsilon_{y}^{A}=c \varepsilon_{y}+\chi
$$

$\left({ }^{7}\right)$ Here we have used the fact that for any $E \subset \mathbb{R}^{n}, c_{\alpha}(E)=0$ if and only if $c_{\alpha}\left(E^{*}\right)=0$, where $E^{*}$ is the $J_{y}$-image of $E \backslash\{y\}$ [11, p. 261]. This also implies that $\nu^{*}$ is $c_{\alpha}$-absolutely continuous whenever $\nu$ is so. 
where $0<c<1$ and $\chi \in \mathfrak{M}^{+}$is a nonzero measure with $\chi(\{y\})=0$, the inequality $c<1$ being clear from (4.6) applied to $\varepsilon_{y}$. But then

$$
|x-y|^{\alpha-n}=U^{\varepsilon_{y}}(x)=U^{\varepsilon_{y}^{A}}(x)=c|x-y|^{\alpha-n}+U^{\chi}(x) \quad \text { n.e. on } A,
$$

and consequently

$$
U^{\chi_{1}}(x)=|x-y|^{\alpha-n} \quad \text { n.e. on } A \text {, where } \chi_{1}:=\chi /(1-c) .
$$

Since $\chi_{1}(\{y\})=0,66.7$ applied to $\chi_{1}$ yields $U^{\left(\chi_{1}\right)^{*}}=1$ n.e. on $A^{*}$, and hence $\left(\chi_{1}\right)^{*} \in \Theta_{A^{*}}$, which in view of Lemma 5.3 again contradicts our hypothesis that $\gamma_{A^{*}}$ does not exist.

THEOREM 6.10. A point $y \in \bar{A}$ is inner $\alpha$-irregular for $A$ if and only if

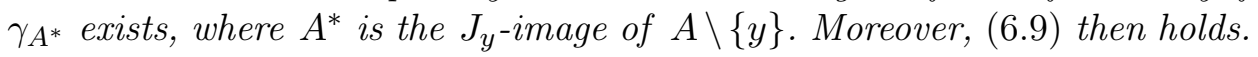

Proof. This follows by combining Lemmas 6.8 and 6.9.

6.2. Proof of Theorem 6.6. We shall first establish the following lemma.

Lemma 6.11. If $\gamma_{A}$ exists, then

$$
U^{\gamma_{A}}=1 \quad \text { on } \bar{A} \backslash A_{I} .
$$

Proof. Fix $x \in \bar{A} \backslash A_{I}$ and $y \in \mathbb{R}^{n}, y \neq x$, and write $r:=|x-y|$. For $E \subset \mathbb{R}^{n}$, let $E^{*}$ denote the $J_{y}$-image of $E \backslash\{y\}$. Then

$$
M^{-1} c_{\alpha}(E) \leq c_{\alpha}\left(E^{*}\right) \leq M c_{\alpha}(E) \quad \text { for every } E \subset B(x, r / 2),
$$

$M \in(1, \infty)$ being independent of $E$ (cf. (6.4)). By the Wiener type criterion, this implies that $x^{*}:=J_{y}(x)$ is inner $\alpha$-regular for $A^{*}$. Hence, by (6.1) applied to $\varepsilon_{y}$

$$
U^{\varepsilon_{y}^{A^{*}}}\left(x^{*}\right)=U^{\varepsilon_{y}}\left(x^{*}\right)=\left|x^{*}-y\right|^{\alpha-n} .
$$

Assume that $\gamma_{A}$ exists. According to Lemma 6.8 with $A$ and $A^{*}$ interchanged,

$$
\gamma_{A}=\mathcal{K}_{y} \varepsilon_{y}^{A^{*}}
$$

and $U^{\gamma_{A}}(x)=1$ is obtained by combining 6.12 with 6.7 applied to $\nu=\varepsilon_{y}^{A^{*}}$.

Write $A^{(k)}:=A \cap B(0, k)$. Theorem 6.6 will be established once we show

$$
c_{\alpha}\left(A_{I} \cap A^{(k)}\right)=0 \quad \text { for every } k \in \mathbb{N} \text {. }
$$

By Lemma 6.11 applied to $A^{(k)}, U^{\gamma_{A}(k)}=1$ on $\overline{A^{(k)}} \backslash A_{I}^{(k)}$. Comparing this with 5.1 applied to $A^{(k)}$ implies that $A_{I}^{(k)} \cap A^{(k)}$ has inner capacity zero. But it is clear from the Wiener type criterion that $A_{I} \cap A^{(k)}=A_{I}^{(k)} \cap A^{(k)}$, and 6.13) follows. 
7. Description of $U^{\gamma_{A}}$ and $S\left(\gamma_{A}\right)$ for $A$ closed. Define the reduced kernel $\breve{A}$ [11, p. 164] of $A$ as the set of all $x \in A$ such that

$$
c_{\alpha}(B(x, r) \cap A)>0 \quad \text { for any } r>0 .
$$

Assuming that $A$ is closed and $\gamma_{A}$ exists, we provide in Theorem 7.2 below a detailed description of $U^{\gamma_{A}}$ and $S\left(\gamma_{A}\right)$. When doing this, we can assume without any loss of generality that $A=\breve{A}$. Indeed, $\breve{A}$ is closed along with $A$. Furthermore, since $c_{\alpha}(A \backslash \breve{A})=0, \gamma_{A}$ serves simultaneously as $\gamma_{\breve{A}}$, and also $A_{I}=(\breve{A})_{I}$.

LEMmA 7.1. Under these hypotheses, assume moreover that $\alpha=2$. Then there is a unique connected component $\Delta$ of the (open) set $A^{c}$ such that $\gamma_{\tilde{A}}$ exists, where

$$
\tilde{A}:=\Delta^{c} \quad(\supset A) .
$$

Proof. For $A$ compact, $\Delta$ is in fact the (unique) unbounded connected component of $A^{c}$. For $A$ noncompact, fix any $y \in A^{c}$ and consider the $J_{y^{-}}$ image $K_{y}$ of $\mathrm{Cl}_{\overline{\mathbb{R}^{n}}} A$. Since $\gamma_{A}$ exists, Theorem 6.10 with $A$ and $A^{*}$ interchanged shows that $y$ is a 2-irregular point of $K_{y}$. By [4, Chapter VIII, Section 6, Remark], there is therefore a unique connected component $D$ of the (open) set $K_{y}^{c}$ such that $y$ is 2 -irregular for $D^{c}$, and the $J_{y}$-image $\Delta$ of this $D$ is as claimed.

THEOREM 7.2. Under the above mentioned hypotheses and notation, (5.1) and (5.2) can be specified as follows: if $\alpha<2$, then

$$
\begin{array}{ll}
U^{\gamma_{A}}=1 & \text { on } A \backslash A_{I}, \\
U^{\gamma_{A}}<1 & \text { on } A^{c},
\end{array}
$$

while for $\alpha=2\left({ }^{8}\right)$,

$$
\begin{array}{ll}
U^{\gamma_{A}}=1 & \text { on } \tilde{A} \backslash \tilde{A}_{I}, \\
U^{\gamma_{A}}<1 & \text { on } \tilde{A}^{c} .
\end{array}
$$

Furthermore,

$$
S\left(\gamma_{A}\right)= \begin{cases}A & \text { if } \alpha<2, \\ \partial \tilde{A} & \text { if } \alpha=2 .\end{cases}
$$

Proof. Assume first that $\alpha<2$. Noting that (7.1) has been established in Lemma 6.11, we first prove

$$
U^{\gamma_{A}}<1 \quad \text { on } S\left(\gamma_{A}\right)^{c} .
$$

$\left({ }^{8}\right)$ Observe that $\tilde{A}_{I} \subset A_{I}$, which is seen from Corollary 6.5 and the inclusion $\partial \tilde{A} \subset \partial A$. 
Suppose that this fails for some $x_{0} \in S\left(\gamma_{A}\right)^{c}$. Then, according to 5.2),

$$
U^{\gamma_{A}}\left(x_{0}\right)=1 \text {. }
$$

Choose $\varepsilon>0$ so that $\bar{B}\left(x_{0}, \varepsilon\right) \subset S\left(\gamma_{A}\right)^{c}$. Since $U^{\gamma_{A}}$ is $\alpha$-harmonic on $B\left(x_{0}, \varepsilon\right)$ [11. Chapter I, Section $6, \mathrm{n}^{\circ} 20$ ] and continuous on $\bar{B}\left(x_{0}, \varepsilon\right)$, we conclude from (5.2) and (7.7) with the aid of [11, Theorem 1.28] that $U^{\gamma_{A}}=1$ a.e. on $\mathbb{R}^{n}$. By the definition of $\alpha$-superharmonicity, this yields $U^{\gamma_{A}}=1$ on $\mathbb{R}^{n}$. Hence, $\gamma_{A}$ serves as an inner equilibrium measure on the whole of $\mathbb{R}^{n}$, which is impossible (e.g. by Theorem 5.5).

To prove the former equality in 7.5), suppose to the contrary that there is $x_{1} \in A$ such that $x_{1} \notin S\left(\gamma_{A}\right)$, and consider an open neighborhood $V \subset$ $S\left(\gamma_{A}\right)^{c}$ of $x_{1}$. In view of (7.6), $U^{\gamma_{A}}<1$ on $V$. On the other hand, since $c_{\alpha}(V \cap A)>0$ because of the assumption $A=\breve{A}$, we see from (5.1) that $U^{\gamma_{A}}\left(x_{2}\right)=1$ for some $x_{2} \in V \cap A$. The contradiction obtained shows that, indeed, $S\left(\gamma_{A}\right)=A$. Substituting this equality into (7.6) establishes (7.2).

In the rest of the proof, $\alpha=2$. We first establish (7.4) and the latter relation in (7.5) for $\gamma_{\tilde{A}}$ in place of $\gamma_{A}$. (Relation 7.3 with $\gamma_{\tilde{A}}$ in place of $\gamma_{A}$ holds according to Lemma 6.11) Suppose that $(7.4)$ fails for some $x_{3} \in \tilde{A}^{c}$. By 5.2 applied to $\tilde{A}$, the function $U^{\gamma} \tilde{A}$ then takes its maximum value 1 at $x_{3}$, and hence everywhere on $\tilde{A}^{c}, U^{\gamma} \tilde{A}$ being harmonic on the domain $\tilde{A}^{c}$. This combined with (5.1) gives $U^{\gamma} \tilde{A}=1$ n.e. on $\mathbb{R}^{n}$, which is impossible (e.g. by Theorem 5.5).

By use of [11, Theorem 1.12], we observe from (5.1) applied to $\tilde{A}$ that the restriction of $\gamma_{\tilde{A}}$ to the interior of $\tilde{A}$ equals 0 , and so $S\left(\gamma_{\tilde{A}}\right) \subset \partial \tilde{A}$. For the converse, suppose to the contrary that there is $x_{4} \in \partial \tilde{A}$ such that $x_{4} \notin S\left(\gamma_{\tilde{A}}\right)$. Choose an open neighborhood $V_{1}$ of $x_{4}$ so that $V_{1} \cap S\left(\gamma_{\tilde{A}}\right)=\emptyset$. Since $c_{2}\left(V_{1} \cap \tilde{A}\right)>0, U^{\gamma} \tilde{A}$ takes the value 1 at some point in $V_{1}$, and hence everywhere on $V_{1}$, again by the maximum principle. This contradicts (7.4).

The proof is completed by noting that $\gamma_{A}=\gamma_{\tilde{A}}$. Indeed, as $\partial \tilde{A} \subset A \subset \tilde{A}$, both $\gamma_{A}$ and $\gamma_{\tilde{A}}$ are supported by $A$ and have the potentials equal to 1 n.e. on $A$. Being $c_{\alpha}$-absolutely continuous by Lemma 5.3 , these measures must be equal according to Corollary 5.4.

8. Integral representation of inner swept measure and applications. Throughout this section we assume that

$$
\Omega:=\bar{A}^{c} \neq \emptyset .
$$

8.1. Integral representation of inner swept measure. Lemma 8.1 and Theorem 8.2 below strengthen [10, Lemma 3.16, Theorem 3.17], dealing with balayage onto closed sets. For the notion of a $\mu$-adequate family of measures, see [3, Chapter V, Section 3, $\mathrm{n}^{\circ} 1$, Definition 1]. 
LEMma 8.1. For every $\mu \in \mathfrak{M}_{\Omega}^{+}$, the family $\left(\varepsilon_{y}^{A}\right)_{y \in \Omega}$ is $\mu$-adequate; that is,

(a) for any $f \in C_{0}\left(\mathbb{R}^{n}\right)$, the function $y \mapsto \int f d \varepsilon_{y}^{A}$ is $\mu$-integrable on $\Omega$,

(b) the map $y \mapsto \varepsilon_{y}^{A}$ is vaguely $\mu$-measurable on $\Omega$.

Proof. The proof of (a) repeats word-for-word the proof of Lemma 3.16(a) in [10, except for applying our relations (3.10) and 4.6 instead of [10, (3.11), (3.18)], the concept of balayage being now understood as described in Section 3 above. Since $U^{\mu^{A}}$ is finite and continuous on $\Omega$, the inner swept measure $\mu^{A}$ being supported by $\bar{A}$, (b) can likewise be obtained by an adaptation of [10, proof of Lemma 3.16(b)].

THEOREM 8.2. For any $\mu \in \mathfrak{M}_{\Omega}^{+}$, the integral representation 1.1 holds.

Proof. Fix $\mu \in \mathfrak{M}_{\Omega}^{+}$. Since the family $\left(\varepsilon_{y}^{A}\right)_{y \in \Omega}$ is $\mu$-adequate, according to [3, Chapter $\mathrm{V}$, Section 3, $\mathrm{n}^{\circ} 2$ ] we can define the Radon measure $\nu=$ $\int \varepsilon_{y}^{A} d \mu(y)$ on $\mathbb{R}^{n}$ by means of the formula

$$
\int f(z) d \nu(z)=\int\left(\int f(z) d \varepsilon_{y}^{A}(z)\right) d \mu(y) \quad \text { for every } f \in C_{0}\left(\mathbb{R}^{n}\right) .
$$

According to [3, Chapter V, Section 3, Proposition 1], this identity remains valid when $f$ is allowed to be any positive l.s.c. function on $\mathbb{R}^{n}$. For given $x \in \mathbb{R}^{n}$ we apply this to $f(z)=|x-z|^{\alpha-n}, z \in \mathbb{R}^{n}$ :

$$
U^{\nu}(x)=\int\left(\int|x-z|^{\alpha-n} d \varepsilon_{y}^{A}(z)\right) d \mu(y)=\int U^{\varepsilon_{y}^{A}}(x) d \mu(y) .
$$

To establish 1.1, it remains to show that $\nu=\mu^{A}$, or equivalently (cf. Definition 3.9

$$
E(\nu, \lambda)=E\left(\mu, \lambda^{A}\right) \quad \text { for every } \lambda \in \mathcal{E}^{+} .
$$

Applying 3.10 with $\mu=\varepsilon_{y}$ and 8.1 , by Fubini's theorem we get

$$
\begin{aligned}
E(\nu, \lambda) & =\int U^{\nu}(x) d \lambda(x)=\int\left(\int U^{\varepsilon_{y}^{A}}(x) d \mu(y)\right) d \lambda(x) \\
& =\int\left(\int U^{\varepsilon_{y}^{A}}(x) d \lambda(x)\right) d \mu(y)=\int\left(\int U^{\varepsilon_{y}}(x) d \lambda^{A}(x)\right) d \mu(y) \\
& =\int\left(\int|x-y|^{\alpha-n} d \mu(y)\right) d \lambda^{A}(x)=\int U^{\mu} d \lambda^{A}=E\left(\mu, \lambda^{A}\right),
\end{aligned}
$$

which is the required identity.

Corollary 8.3. For any $\mu \in \mathfrak{M}_{\Omega}^{+}, \mu^{A}$ is $c_{\alpha}$-absolutely continuous.

Proof. Consider a compact set $K \subset \bar{A}$ with $c_{\alpha}(K)=0$. For every $y \in \Omega$, the swept measure $\varepsilon_{y}^{A}$ has finite energy (see Remark 6.2), and hence $\varepsilon_{y}^{A}(K)=0$. Applying [3, Chapter V, Section 3, Theorem 1] we obtain, by (1.1),

$$
\int 1_{K} d \mu^{A}=\int d \mu(y) \int 1_{K}(x) d \varepsilon_{y}^{A}(x)=0,
$$

and hence $\mu^{A}$ is indeed $c_{\alpha}$-absolutely continuous. 
Corollary 8.4. For any $\mu \in \mathfrak{M}_{\Omega}^{+}$and $A$ closed, $\mu^{A}$ is uniquely determined by 3.1 among the $c_{\alpha}$-absolutely continuous measures of the class $\mathfrak{M}_{A}^{+}$.

Proof. This follows from Corollary 8.3 in view of [11, p. 178, Remark].

8.2. Description of the support of the inner swept measure. Let $A$ be closed and $\mu \in \mathfrak{M}_{\Omega}^{+}$. To establish a description of $S\left(\mu^{A}\right)$, we assume that $A$ coincides with its reduced kernel $\breve{A}$, while $\mu$ is carried by a connected component $\Omega_{0}$ of $\Omega$. This involves no loss of generality, as can be seen from (3.14) and 4.5) (with $A^{\prime}=\breve{A}$ ).

THEOREM 8.5. Under these hypotheses,

$$
S\left(\mu^{A}\right)= \begin{cases}A & \text { if } \alpha<2, \\ \partial \Omega_{0} & \text { if } \alpha=2 .\end{cases}
$$

Proof. For any $y \in \Omega_{0}$, we denote by $K_{y}$ the $J_{y^{-i m a g e}}$ of $\mathrm{Cl}_{\overline{\mathbb{R}^{n}}} A$, and by $\gamma_{K_{y}}$ the equilibrium measure on the (compact) set $K_{y}$. Since the $J_{y}$-image of any $E \subset A$ with $c_{\alpha}(E)=0$ again has zero inner capacity (cf. footnote 7), $K_{y}$ coincides with its reduced kernel. Thus, $S\left(\gamma_{K_{y}}\right)=K_{y}$ for $\alpha<2$, while for $\alpha=2, S\left(\gamma_{K_{y}}\right)$ coincides with the outer boundary of $K_{y}$, that is, the boundary of the unbounded connected component of $K_{y}^{c}$ (see 7.5 ) or [11, Chapter II, Section 3, $\mathrm{n}^{\circ}$ 13]). Now applying the integral representation (1.1) (which holds under the stated hypotheses, see Theorem 8.2) and the fact that for every $y \in \Omega_{0}, \varepsilon_{y}^{A}$ is the Kelvin transform of $\gamma_{K_{y}}$ (see Lemma 6.8, we obtain 8.2 .

8.3. Further criteria for the existence of the inner equilibrium measure. Finally, we provide necessary and sufficient conditions for the existence of $\gamma_{A}$, given in terms of $\mu^{A}\left(\mathbb{R}^{n}\right)$ with $\mu$ suitably chosen. Since $\gamma_{A}$ and $\gamma_{\breve{A}}$ exist or do not exist simultaneously, we can certainly assume that $A=\breve{A}$.

TheOREM 8.6. $\gamma_{A}$ exists if there is a measure $\mu \in \mathfrak{M}_{\Omega}^{+}$with

$$
\mu^{A}\left(\mathbb{R}^{n}\right)<\mu\left(\mathbb{R}^{n}\right) .
$$

Proof. Assume that 8.3 holds for some $\mu \in \mathfrak{M}_{\Omega}^{+}$, and suppose on the contrary that $\gamma_{A}$ does not exist. Fix $y \in \Omega$ and consider the $J_{y}$-image $A^{*}$ of $A$; then, according to Lemma 6.9 with $A$ and $A^{*}$ interchanged, $y$ is inner $\alpha$-regular for $A^{*}$. According to Lemma 6.11, this gives

$$
U^{\gamma_{A^{*}}}(y)=1 \text {. }
$$

(Note that an inner equilibrium measure $\gamma_{A^{*}}$ exists, $A^{*}$ being relatively compact.) On the other hand, for the Kelvin transform $\left(\gamma_{A^{*}}\right)^{*}=\mathcal{K}_{y} \gamma_{A^{*}}$ of $\gamma_{A^{*}}$, according to Lemma 6.8 we have

$$
\left(\gamma_{A^{*}}\right)^{*}=\varepsilon_{y}^{A} .
$$


Therefore, applying (6.6) to $\nu=\varepsilon_{y}^{A}$, in view of 8.4 we get

$$
\varepsilon_{y}^{A}\left(\mathbb{R}^{n}\right)=U^{\gamma_{A^{*}}}(y)=1
$$

Substituting this now into (1.1) (which holds according to Theorem 8.2) and applying [3, Chapter V, Section 3, Theorem 1], we obtain

$$
\mu^{A}\left(\mathbb{R}^{n}\right)=\int 1 d \mu^{A}=\int d \mu(y) \int 1(x) d \varepsilon_{y}^{A}(x)=\int 1 d \mu=\mu\left(\mathbb{R}^{n}\right),
$$

which, however, contradicts 8.3 .

For $A$ closed, Theorem 8.6 can be reversed.

THEOREM 8.7. For A closed, $\gamma_{A}$ exists if and only if 8.3 holds for some $\mu \in \mathfrak{M}_{\Omega}^{+}$. Actually, if $\gamma_{A}$ exists, then 8.3 holds for every nonzero $\mu \in \mathfrak{M}_{\Omega_{\alpha}}^{+}$, where

$$
\Omega_{\alpha}:= \begin{cases}\Omega & \text { if } \alpha<2, \\ \Delta & \text { if } \alpha=2,\end{cases}
$$

$\Delta$ being defined in Lemma 7.1 .

Proof. In view of Theorem 8.6, it is enough to establish the latter part of the theorem. Assume $\gamma_{A}$ exists and fix any nonzero $\mu \in \mathfrak{M}_{\Omega_{\alpha}}^{+}$. By 7.2 and 7.4 ,

$$
U^{\gamma_{A}}<1 \quad \text { on } \Omega_{\alpha} .
$$

Since both $\gamma_{A}$ and $\mu^{A}$ are $c_{\alpha}$-absolutely continuous (see Lemma 5.3 and Corollary 8.3, respectively) and supported by $A$, from (5.1), (3.1), and (8.5) we obtain

$\mu^{A}\left(\mathbb{R}^{n}\right)=\int U^{\gamma_{A}} d \mu^{A}=\int U^{\mu^{A}} d \gamma_{A}=\int U^{\mu} d \gamma_{A}=\int U^{\gamma_{A}} d \mu<\mu\left(\Omega_{\alpha}\right)=\mu\left(\mathbb{R}^{n}\right)$,

which is 8.3$)$.

ExAmple 8.8. Let $n=3, \alpha=2$, and let $A$ be the rotation body defined by (5.8). Then

$$
\mu^{A}\left(\mathbb{R}^{3}\right)=\mu\left(\mathbb{R}^{3}\right) \quad \text { for every } \mu \in \mathfrak{M}_{A^{c}}^{+}
$$

provided that $\varrho$ in $(5.8)$ is given by $(5.9)$, and

$$
\mu^{A}\left(\mathbb{R}^{3}\right)<\mu\left(\mathbb{R}^{3}\right) \quad \text { for every nonzero } \mu \in \mathfrak{M}_{A^{c}}^{+}
$$

whenever (5.10) or 5.11) holds (see Figure 1). This follows by combining Theorems 8.6, 8.7 and Corollary 4.9 with Example 5.8.

Acknowledgements. I thank Bent Fuglede for going through the entire manuscript thoroughly and constructively. 


\section{References}

[1] J. Bliedtner and W. Hansen, Potential Theory. An Analytic and Probabilistic Approach to Balayage, Springer, Berlin, 1986.

[2] N. Bourbaki, General Topology, Chapters 1-4, Springer, Berlin, 1989.

[3] N. Bourbaki, Integration, Chapters 1-6, Springer, Berlin, 2004.

[4] M. Brelot, Éléments de la Théorie Classique du Potentiel, Les Cours Sorbonne, Paris, 1961.

[5] M. Brelot, On Topologies and Boundaries in Potential Theory, Lecture Notes in Math. 175, Springer, Berlin, 1971.

[6] H. Cartan, Théorie générale du balayage en potentiel newtonien, Ann. Univ. Grenoble 22 (1946), 221-280.

[7] J. L. Doob, Classical Potential Theory and Its Probabilistic Counterpart, Springer, Berlin, 1984.

[8] R. E. Edwards, Functional Analysis. Theory and Applications, Holt, Rinehart and Winston, New York, 1965.

[9] B. Fuglede, On the theory of potentials in locally compact spaces, Acta Math. 103 (1960), 139-215.

[10] B. Fuglede and N. Zorii, Green kernels associated with Riesz kernels, Ann. Acad. Sci. Fenn. Math. 43 (2018), 121-145.

[11] N. S. Landkof, Foundations of Modern Potential Theory, Springer, Berlin, 1972.

[12] M. Riesz, Intégrales de Riemann-Liouville et potentiels, Acta Sci. Math. Univ. Szeged 9 (1938), 1-42.

Natalia Zorii

Institute of Mathematics

Academy of Sciences of Ukraine

Tereshchenkivska 3

01601 Kyiv, Ukraine

ORCID: 0000-0003-3228-8654

E-mail: natalia.zorii@gmail.com 
